\title{
Theoretical Investigations of $I=5 / 2$ Quadrupolar Spin Dynamics in the Sudden-Passage Regime
}

\author{
Jamie D. Walls, Kwang Hun Lim, John W. Logan, Jeffry T. Urban, Alexej Jerschow*, and \\ Alexander Pines ${ }^{\dagger}$ \\ Materials Sciences Division, Lawrence Berkeley National Laboratory, Berkeley, California 94720 \\ and Department of Chemistry, University of California, Berkeley, California 94720
}

\begin{abstract}
The theoretical approach utilizing bimodal Floquet theory in the quadrupolar/central-transition interaction frame, presented in an earlier article [J. D. Walls, K. H. Lim, and A. Pines, J. Chem. Phys. 116, 79 (2002)], is extended to describe the more complicated spin dynamics of $I=5 / 2$ spin systems. Rotary resonance effects occur when the strength of the radiofrequency irradiation, $\omega_{1}$, matches the sample spinning speed, $\omega_{r}$, at the conditions $\omega_{1}=\frac{2}{3} n \omega_{r}$ ( $n$ integral). At these conditions, conversions of both triple-quantum and five-quantum coherences to central-quantum coherence are observed. Between rotary resonance conditions $\left(\frac{2 n}{3} \omega_{r}<\omega_{1}<\frac{2(n+1)}{3} \omega_{r}\right)$, five-quantum as well as triple-quantum coherences can be created from equilibrium $z$-magnetization via a nutation mechanism. In addition, effective transfer between five-quantum and triple-quantum coherences also is observed in between rotary resonance conditions. These effects have been investigated theoretically and verified by both numerical calculations and experimental results.
\end{abstract}

\footnotetext{
*Present address: New York University, Chemistry Department, New York, NY 10003

${ }^{\dagger}$ Corresponding author. email: pines@cchem.berkeley.edu, fax: (1) 510-486-5744
} 


\section{INTRODUCTION}

Approximately sixty percent of all NMR-active nuclei are quadrupolar $(I>1 / 2)$, and due to the high sensitivity of the nuclear quadrupole moment to local electric field gradients, NMR studies of quadrupolar nuclei have been of great importance in the study of glasses, minerals, zeolites, and other inorganic materials in the solid state ${ }^{1-8}$. In high magnetic fields, the first-order quadrupolar interaction has no effect on the frequency of the spectrum's central transition (CT), and its effect may be removed from the satellite transitions via rotor-synchronized magic-angle spinning (MAS). However, due to the fact that the quadrupole coupling is in most cases a large perturbation to the Zeeman energy, the second-order quadrupolar coupling is non-negligible and broadens the lineshapes, thereby limiting the resolution and sensitivity of the NMR experiment. The early dynamic angle spinning $(\mathrm{DAS})^{9,10}$ and double rotation $(\mathrm{DOR})^{11}$ experiments were developed in order to average away the second-order quadrupolar interaction by purely mechanical means, by spinning the sample about more than one axis. Frydman et al. ${ }^{12}$ subsequently proposed the MQMAS technique, which refocuses the anisotropic second-order quadrupolar interaction by evolving under various multiple-quantum coherences while magic-angle sample spinning. The necessity of evolving under various multiple-quantum coherences requires the design of pulse sequences that are capable of producing the desired excitation and transfers of these coherences in the presence of a large, time-dependent first-order quadrupolar interaction. Therefore, in order to design RF pulses to manipulate quadrupolar nuclei for MQMAS spectroscopy, an understanding of the spin dynamics under both RF irradiation and MAS is required.

A. Vega demonstrated that the spin-locking behavior of quadrupolar nuclei under MAS conditions is drastically different from that of $I=1 / 2$ nuclei $^{13,14}$. The theory of the spin dynamics for $I=1 / 2$ nuclei is relatively well established due to the fact that RF field strength is usually much larger than the time-dependent spin interactions (such as dipoledipole coupling and chemical shift anisotropy), and transforming the Hamiltonian into the 
RF interaction frame yields valuable insight. However, the situation becomes quite different for quadrupolar spin systems $(I>1 / 2)$, since the time-dependent quadrupolar interaction typically is much larger than the time-independent RF field strength under most practical experimental conditions, resulting in unique quadrupolar spin dynamics. The behavior of quadrupolar systems has been found to depend strongly on the relative magnitudes of the RF irradiation, $\omega_{1}$, the sample spinning frequency, $\omega_{r}$, and the quadrupolar frequency, $\omega_{Q}$. An adiabaticity parameter, $\alpha=\frac{\omega_{1}^{2}}{\omega_{r} \omega_{Q}}$, was introduced by Vega in order to characterize the spin-locking dynamics. ${ }^{13}$ In the adiabatic regime $(\alpha \gg 1)$, where the $\mathrm{RF}$ power is high and the spinning speed is low, CT coherences are transferred to multiple-quantum coherences as $\omega_{Q}$ undergoes zero-crossings during the course of the MAS. The quadrupolar spin dynamics in the sudden-passage regime $(\alpha \ll 1)$, where the $\mathrm{RF}$ power is low and the spinning speed is high, is markedly different from the dynamics in the adiabatic regime. In Vega's treatment, no transfers between CT and MQ coherences are predicted in the sudden-passage limit, and thus the CT coherence is efficiently spin-locked for all times during the irradiation. This semiquantitive physical picture has explained successfully some of the main features of the spin dynamics that have been observed in experiments, such as the coherence transfers in the adiabatic regime ${ }^{15}$ and efficient spin-locking at certain RF values in the sudden-passage limit. However, it has been observed that when the RF power is appropriately matched to the spinning speed at the conditions $\omega_{1}=\frac{4 n \omega_{r}}{2 I+1}$ (where $n$ is a positive integer), the CT coherence does not remain spin-locked. ${ }^{16,17}$ Additionally, similar resonant effects were observed in homonuclear dipolar recoupling experiments involving quadrupolar nuclei under MAS conditions. ${ }^{18}$ Also, Vosegaard et al. ${ }^{19}$ reported unusual spin dynamics for $I=3 / 2$ nuclei in the sudden-passage regime. At the conditions $\omega_{1}=n \omega_{r}$, an efficient conversion between CT and triple-quantum coherences occurs in the sudden-passage limit, whereas the excitation efficiency of triple-quantum coherence from $z$-magnetization is enhanced when in between the resonant conditions at $\frac{n}{2} \omega_{r}<\omega_{1}<\frac{n+1}{2} \omega_{r}$. We have developed recently a new theoretical approach in order to explain the interesting spin dynamics in $I=3 / 2$ 
systems using bimodal Floquet theory in a novel interaction frame that combines the CT RF and first-order quadrupolar interactions. ${ }^{20}$ We demonstrated that the basis for the "rotary resonance" (RR) effects at $\omega_{1}=n \omega_{r}$ was the result of a degenerate mixing of the triple-

quantum coherence states $|T \pm\rangle=\frac{1}{\sqrt{2}}(|3 / 2\rangle \pm|-3 / 2\rangle)$ with the CT coherence states $|C \pm\rangle=$ $\frac{1}{\sqrt{2}}(|1 / 2\rangle \pm|-1 / 2\rangle)$ at the conditions $\omega_{1}=n \omega_{r}$, whereas triple-quantum excitation was found to result from higher-order terms in the Hamiltonian.

In this paper, we extend our previous study of the spin dynamics of quadrupolar nuclei in the sudden-passage regime to $I=5 / 2$ nuclei. We attempt to gain insight into the complicated spin dynamics by again developing a bimodal Floquet treatment using an interaction frame that combines both the CT RF Hamiltonian with the first-order quadrupolar Hamiltonian, only this time evaluating the Floquet Hamiltonian using an effective Hamiltonian treatment. ${ }^{21}$ This theoretical formalism is then compared to exact numerical simulations. Finally, preliminary experimental data are presented on five-quantum to triple-quantum conversion and triple-quantum to single-quantum conversion in the $I=5 / 2{ }^{27} \mathrm{Al}$ system.

\section{THEORY}

The Hamiltonian for a spin $I=5 / 2$ quadrupolar nucleus in the rotating frame under MAS and RF irradiation can be written in a fictitious spin-1/2 operator basis ${ }^{22,23}$ as

$$
\begin{aligned}
H(t)= & H_{Q}^{(1)}(t)+H_{R F} \\
= & \omega_{Q}(t)\left[20 I_{Z}^{1-2}+16 I_{Z}^{2-3}-16 I_{Z}^{4-5}-20 I_{Z}^{5-6}\right]+3 \omega_{1} I_{X}^{3-4} \\
& +\sqrt{5} \omega_{1}\left[I_{X}^{1-2}+I_{X}^{5-6}\right]+2 \sqrt{2} \omega_{1}\left[I_{X}^{2-3}+I_{X}^{4-5}\right],
\end{aligned}
$$

where $\omega_{1}$ is the strength of the RF field and $\omega_{r}$ is the rotor frequency. $H_{Q}^{(1)}(t)$ describes the first-order quadrupolar interaction, and $H_{R F}$ describes the radiofrequency irradiation. The states are labelled by $|1\rangle=\left|m_{I}=+5 / 2\right\rangle,|2\rangle=|+3 / 2\rangle,|3\rangle=|+1 / 2\rangle,|4\rangle=$ $|-1 / 2\rangle,|5\rangle=|-3 / 2\rangle$, and $|6\rangle=|-5 / 2\rangle$. The effects of the second-order quadrupolar 
interaction, chemical shift anisotropy (CSA), and resonance offset terms will be discussed later in the Article. The time-dependent first-order quadrupolar coupling is given by:

$$
\omega_{Q}(t)=\omega_{Q}\left[C_{1} \cos \left(\omega_{r} t+\gamma\right)+C_{2} \cos \left(2 \omega_{r} t+2 \gamma\right)+S_{1} \sin \left(\omega_{r} t+\gamma\right)+S_{2} \sin \left(2 \omega_{r} t+2 \gamma\right)\right],
$$

where $C_{1}, C_{2}, S_{1}$, and $S_{2}$ depend on the angle $\theta$ between the rotor axis and the Zeeman field axis, and on the crystallite-dependent Euler angles $[\alpha, \beta, \gamma]$ that relate the quadrupolar principal axis system (PAS) to the rotor-fixed coordinate system. Expressions for $\omega_{Q}, C_{1}, C_{2}, S_{1}$, and $S_{2}$ are given in Appendix A.

The propagator for the Hamiltonian is:

$$
U(t)=T\left\{\exp \left[-i \int_{0}^{t} H\left(t^{\prime}\right) d t^{\prime}\right]\right\}
$$

where $T$ is the Dyson time-ordering operator. The evaluation of this propagator is difficult due to the fact that in general $\left[H(t), H\left(t^{\prime}\right)\right] \neq 0$ for times $t \neq t^{\prime}$. We previously introduced a transformation into an interaction frame defined by the first-order quadrupolar Hamiltonian and the CT operator from the RF Hamiltonian that was found to be useful in describing the spin dynamics in a bimodal Floquet theory treatment. ${ }^{20}$ Although such a treatment will be used in the following discussion, it was shown recently ${ }^{24}$ for the $I=3 / 2$ system that unimodal Floquet theory also can give the same results (the equivalence between the two approaches is demonstrated in Appendix B). The quadrupolar/CT transformation for a spin $I=5 / 2$ is:

$$
W(t)=T\left\{\exp \left[-i \int_{0}^{t} d t^{\prime}\left(H_{Q}^{(1)}\left(t^{\prime}\right)+3 \omega_{1} I_{X}^{3-4}\right)\right]\right\} .
$$

This transformation can be evaluated easily since the CT Hamiltonian commutes with the first-order quadrupolar Hamiltonian. The Hamiltonian in this interaction frame is given by:

$$
\begin{aligned}
H_{\mathrm{INT}}(t)= & W^{\dagger}(t) H(t) W(t)-i W^{\dagger}(t) \frac{d W(t)}{d t} \\
= & \sqrt{5} \omega_{1}\left[\left(I_{X}^{1-2}+I_{X}^{5-6}\right) \cos \left(12 \int_{0}^{t} \omega_{Q}\left(t^{\prime}\right) d t^{\prime}\right)+\left(I_{Y}^{5-6}-I_{Y}^{1-2}\right) \sin \left(12 \int_{0}^{t} \omega_{Q}\left(t^{\prime}\right) d t^{\prime}\right)\right] \\
& +2 \sqrt{2} \omega_{1}\left[\left\{\left(I_{X}^{2-3}+I_{X}^{4-5}\right) \cos \left(\frac{3 \omega_{1} t}{2}\right)+\left(I_{Y}^{2-4}-I_{Y}^{3-5}\right) \sin \left(\frac{3 \omega_{1} t}{2}\right)\right\} \cos \left(6 \int_{0}^{t} \omega_{Q}\left(t^{\prime}\right) d t^{\prime}\right)\right]
\end{aligned}
$$




$$
\begin{aligned}
& +2 \sqrt{2}\left[\left\{\left(I_{Y}^{4-5}-I_{Y}^{2-3}\right) \cos \left(\frac{3 \omega_{1} t}{2}\right)+\left(I_{X}^{3-5}+I_{X}^{2-4}\right) \sin \left(\frac{3 \omega_{1} t}{2}\right)\right\} \sin \left(6 \int_{0}^{t} \omega_{Q}\left(t^{\prime}\right) d t^{\prime}\right)\right] \\
= & \sum_{N=-\infty}^{\infty} \sum_{M=0, \pm 1} H_{N, M} \exp \left(-i N \omega_{r} t\right) \exp \left(-i \frac{3 M}{2} \omega_{1} t\right),
\end{aligned}
$$

where

$$
\begin{aligned}
\cos \left(12 \int_{0}^{t} \omega_{Q}\left(t^{\prime}\right) d t^{\prime}\right) & =\sum_{N=-\infty}^{\infty} P_{N} \exp \left(-i N \omega_{r} t\right) \\
\sin \left(12 \int_{0}^{t} \omega_{Q}\left(t^{\prime}\right) d t^{\prime}\right)= & \sum_{N=-\infty}^{\infty} Q_{N} \exp \left(-i N \omega_{r} t\right) \\
\cos \left(6 \int_{0}^{t} \omega_{Q}\left(t^{\prime}\right) d t^{\prime}\right)= & \sum_{N=-\infty}^{\infty} R_{N} \exp \left(-i N \omega_{r} t\right) \\
\sin \left(6 \int_{0}^{t} \omega_{Q}\left(t^{\prime}\right) d t^{\prime}\right)= & \sum_{N=-\infty}^{\infty} S_{N} \exp \left(-i N \omega_{r} t\right) \\
H_{N, \pm 1}= & \sqrt{2} \omega_{1}\left[\left(R_{N}+i S_{N}\right)|T \mp\rangle\left\langle C \mp\left|+\left(R_{N}-i S_{N}\right)\right| C \pm\right\rangle\langle T \pm|\right] \\
H_{N, 0}= & \frac{\sqrt{5}}{2} \omega_{1}\left[\left(P_{N}+i Q_{N}\right)(|q+\rangle\langle T+|+| q-\rangle\langle T-|)\right. \\
& \left.+\left(P_{N}-i Q_{N}\right)(|T+\rangle\langle q+|+| T-\rangle\langle q-|)\right]
\end{aligned}
$$

and $|T \pm\rangle=\frac{1}{\sqrt{2}}(|2\rangle \pm|5\rangle),|C \pm\rangle=\frac{1}{\sqrt{2}}(|3\rangle \pm|4\rangle)$, and $|q \pm\rangle=\frac{1}{\sqrt{2}}(|1\rangle \pm|6\rangle)$ are the triplequantum coherence, central-quantum coherence, and five-quantum coherence subspaces, respectively. The coefficients $Q_{N}, P_{N}, R_{N}$, and $S_{N}$ are dependent on crystallite orientation.

It can be seen from Eq. 5 that the Hamiltonian is modulated at the "natural" frequencies $\omega_{r}$ due to the sample rotation and $\frac{3}{2} \omega_{1}$ due to the CT RF irradiation. The time dependence of $H_{I N T}$ can be removed by a transformation into Floquet space. ${ }^{25,26}$ The price to be paid is that instead of working in a finite-dimensional Hilbert space, calculations must be performed in an infinite-dimensional Floquet space. Since this interaction frame has two natural frequencies, bimodal Floquet theory must be applied. ${ }^{27}$ In Floquet space, the spin states $|C \pm\rangle,|T \pm\rangle,|q \pm\rangle$ become dressed by states $|N, M\rangle$ that are labelled by the number of quadrupolar-induced rotational "quanta" and CT RF "quanta", respectively, yielding the Floquet states $|C \pm, N, M\rangle,|T \pm, N, M\rangle$, and $|q \pm, N, M\rangle$. A Floquet Hamiltonian then can be written as

$$
H_{F}=\omega_{r} N^{r}+\frac{3 \omega_{1}}{2} N^{1}+\left(H_{0,0}+H_{0,1} b^{\dagger}+H_{0,-1} b\right)+H_{0,2}^{\prime}\left(b^{\dagger}\right)^{2}+H_{0,-2}^{\prime} b^{2}
$$




$$
+\sum_{N>0}^{\infty}\left[H_{N, 1}\left(a^{\dagger}\right)^{N} b^{\dagger}+H_{-N, 1} a^{N} b^{\dagger}+H_{N,-1}\left(a^{\dagger}\right)^{N} b+H_{-N,-1} a^{N} b\right]
$$

where the the operators $N^{r}, N^{1}, a^{N},\left(a^{\dagger}\right)^{N}, b^{N}$, and $\left(b^{\dagger}\right)^{N}$ are defined by

$$
\begin{aligned}
\left\langle p, N, M\left|N^{r}\right| p^{\prime}, N^{\prime}, M^{\prime}\right\rangle & =N \delta_{p, p^{\prime}} \delta_{N, N^{\prime}} \delta_{M, M^{\prime}} \\
\left\langle p, N, M\left|N^{1}\right| p^{\prime}, N^{\prime}, M^{\prime}\right\rangle & =M \delta_{p, p^{\prime}} \delta_{N, N^{\prime}} \delta_{M, M^{\prime}} \\
\left\langle p, N, M\left|a^{n}\right| p^{\prime}, N^{\prime}, M^{\prime}\right\rangle & =\delta_{p, p^{\prime}} \delta_{N, N^{\prime}-n} \delta_{M, M^{\prime}} \\
\left\langle p, N, M\left|\left(a^{\dagger}\right)^{n}\right| p^{\prime}, N^{\prime}, M^{\prime}\right\rangle & =\delta_{p, p^{\prime}} \delta_{N, N^{\prime}+n} \delta_{M, M^{\prime}} \\
\left\langle p, N, M\left|b^{n}\right| p^{\prime}, N^{\prime}, M^{\prime}\right\rangle & =\delta_{p, p^{\prime}} \delta_{N, N^{\prime}} \delta_{M, M^{\prime}-n} \\
\left\langle p, N, M\left|\left(b^{\dagger}\right)^{n}\right| p^{\prime}, N^{\prime}, M^{\prime}\right\rangle & =\delta_{p, p^{\prime}} \delta_{N, N^{\prime}} \delta_{M, M^{\prime}+n}
\end{aligned}
$$

The propagator can be written in Hilbert space in terms of the Floquet Hamiltonian as ${ }^{28}$

$$
U(t)=W(t) \sum_{N, M}\left\langle N, M\left|\exp \left(-i H_{F} t\right)\right| 0,0\right\rangle \exp \left[i\left(N \omega_{r}+\frac{3}{2} M \omega_{1}\right) t\right] .
$$

The problem arises as to how to evaluate $\exp \left(-i H_{F} t\right)$. Since $H_{F}$ is infinite-dimensional, exact diagonalization is possible in very few cases; therefore, approximations must be made. Previous techniques have used static perturbation theory to approximate the infinite Floquet Hamiltonian. ${ }^{29,30}$ However, consider dividing Floquet space into an infinite number of three dimensional subspaces $D_{S \pm}^{N, M}$, labelled by $\mathrm{N}$ and $\mathrm{M}$, where $D_{S \pm}^{N, M}=$ $\{|q \pm, N, M\rangle,|T \pm, N, M\rangle,|C \pm, N \mp n, M \pm 1\rangle\}$, as shown in Fig. 1(A). Here $n$ is a positive integer that minimizes

$$
\frac{\left|\Delta \omega^{(0)}\right|}{\sqrt{\left|\Delta \omega^{(0)}\right|^{2}+\left|\left\langle T \pm, N, M\left|H_{F}\right| C \pm, N \mp n, M \pm 1\right\rangle\right|^{2}}}=\frac{\left|n \omega_{r}-\frac{3}{2} \omega_{1}\right|}{\sqrt{\left|n \omega_{r}-\frac{3}{2} \omega_{1}\right|^{2}+2 \omega_{1}^{2}\left|R_{ \pm n}+i S_{ \pm n}\right|^{2}}},
$$

where $\Delta \omega^{(0)}=n \omega_{r}-\frac{3}{2} \omega_{1}$ is the zeroth-order energy difference between $|T \pm, N, M\rangle$ and $|C \pm, N \mp n, M \pm 1\rangle$. Eq. 11 groups together those states that most strongly couple to each other. If the magnitude of the couplings between the various subspaces is less than the energy differences between these subspaces, i.e.,

$$
\begin{aligned}
\mid\left\langle i_{ \pm}^{N, M}\left|H_{F}\right| i_{ \pm}^{N, M}\right\rangle & -\left\langle j_{ \pm}^{N^{\prime}, M^{\prime}}\left|H_{F}\right| j_{ \pm}^{N^{\prime}, M^{\prime}}\right\rangle \mid \\
& =\left|\left(N-N^{\prime}\right) \omega_{r}+\frac{3}{2}\left(M-M^{\prime}\right) \omega_{1}\right|>Z\left|\left\langle i_{ \pm}^{N, M}\left|H_{F}\right| j_{ \pm}^{N^{\prime}, M^{\prime}}\right\rangle\right|
\end{aligned}
$$


for $\left|i_{ \pm}^{N, M}\right\rangle \in D_{S \pm}^{N, M},\left|j_{ \pm}^{N^{\prime}, M^{\prime}}\right\rangle \in D_{S \pm}^{N^{\prime}, M^{\prime}}, N, M \neq N^{\prime}, M^{\prime}$ and $Z>1$, then an "effective Hamiltonian" can be constructed within each subspace. Effective Hamiltonian theory is a perturbative technique that separates the system into different manifolds $D_{S \pm}^{N, M}$ that are weakly coupled to each other, as defined for this case in Eq. 12. The couplings between the various subspaces are treated perturbatively, and the Hamiltonian in each subspace is modified to include the effects of these couplings to any desired order. This technique has been used in optics and atomic physics studies ${ }^{21,31}$ and recently has been applied to the study of homonuclear dipole-coupled spin systems under MAS, with ${ }^{32}$ and without ${ }^{33}$ multiple-pulse homonuclear decoupling. The fraction of crystallites in a randomly oriented powder for which Eq. 12 is satisfied is given in Fig. 2 as a function of $\omega_{1}$ for various values of $Z$. It can be seen that as $\omega_{1}$ increases, the fraction of crystallites for which the effective Hamiltonian treatment is valid decreases, due to the fact that the subspaces $D_{S \pm}^{N, M}$ are no longer weakly coupled.

As shown in Fig. 1(B), an effective Hamiltonian is constructed by generating a Hermitian operator $\widehat{S}$ such that

$$
\exp (i \widehat{S}) H_{F} \exp (-i \widehat{S})=\widehat{H}_{F}
$$

where $\widehat{H}_{F}$ is an infinite-dimensional, block-diagonal matrix. The blocks are labelled by $\widehat{H}_{F}^{N, M}$, which are the effective Hamiltonians in the subspace $D_{S \pm}^{N, M}$. Eq. 13 guarantees that the eigenvalues of $H_{F}$ and $\widehat{H}_{F}$ are identical to each other. Both $\widehat{S}$ and $\widehat{H}_{F}^{N, M}$ can be expanded in orders of the coupling between subspaces as follows: $\widehat{S}=\widehat{S}^{(0)}+\lambda \widehat{S}^{(1)}+\ldots$ and $\widehat{H}_{F}^{N, M}=\widehat{H}_{N, M}^{(0)}+\lambda \widehat{H}_{N, M}^{(1)}+\lambda^{2} \widehat{H}_{N, M}^{(2)}+\ldots$, where $\lambda$ keeps track of the order of the perturbation and is set equal to one in the actual calculation. An effective Hamiltonian can be written within $D_{S \pm}^{N, M}$ to second order in $\lambda$ as

$$
\widehat{H}_{F}^{N, M}=\left(N \omega_{r}+\frac{3 M}{2} \omega_{1}\right) \widehat{1}+
$$




$$
\left(\begin{array}{cccccc}
V_{q+, q+}^{(2)} & V_{q+, T+}^{(1)} & V_{q+, C+}^{(2)} & 0 & 0 & 0 \\
V_{T+, q+}^{(1)} & V_{T+, T+}^{(2)} & V_{T+, C+}^{(1)} & 0 & 0 & 0 \\
V_{C+, q+}^{(2)} & V_{C+, T+}^{(1)} & V_{C+, C+}^{(2)}-\Delta \omega^{(0)} & 0 & 0 & 0 \\
0 & 0 & 0 & V_{C-, C-}^{(2)}+\Delta \omega^{(0)} & V_{C-, T-}^{(1)} & V_{C-, q-}^{(2)} \\
0 & 0 & 0 & V_{T-, C-}^{(1)} & V_{T-, T-}^{(2)} & V_{T-, q-}^{(1)} \\
0 & 0 & 0 & V_{q-, C-}^{(2)} & V_{q-, T-}^{(1)} & V_{q-, q-}^{(2)}
\end{array}\right)
$$

where

$$
\begin{aligned}
S^{(0)} & =\widehat{1} \\
S^{(1)} & =-i \lambda\left[\sum_{j \in D_{S \pm}^{N, M}} \sum_{k \notin D_{S \pm}^{N, M}} \frac{|k\rangle\left\langle j\left|\left\langle k\left|H_{F}\right| j\right\rangle-\right| j\right\rangle\langle k|\left\langle j\left|H_{F}\right| k\right\rangle}{E_{j}-E_{k}}\right] \\
V_{i, j}^{(1)} & =\lambda\left\langle i\left|H_{F}\right| j\right\rangle \\
V_{i, j}^{(2)} & =\frac{\lambda^{2}}{2} \sum_{k \notin D_{S \pm}^{N, M}}\left\langle i\left|H_{F}\right| k\right\rangle\left\langle k\left|H_{F}\right| j\right\rangle\left[\frac{1}{E_{j}-E_{k}}+\frac{1}{E_{i}-E_{k}}\right]
\end{aligned}
$$

Explicit expressions for the various $V_{i, j}^{(1)}$ and $V_{i, j}^{(2)}$ elements are given in Appendix C. Since $D_{S+}^{N, M}$ is not coupled to $D_{S-}^{N, M}$ within $H_{F}^{N, M}$, the effective Hamiltonian reduces to two uncoupled three-state systems. (For the $I=3 / 2$ case, the problem reduced to two uncoupled twostate systems: $\{|T+, N, M\rangle,|C+, N-n, M+1\rangle\}$ and $\left.\{|T-, N, M\rangle,|C-, N+n, M-1\rangle\} .{ }^{20}\right)$

\section{A. Near RR conditions}

Near the RR conditions $\omega_{1}=\frac{2 n}{3} \omega_{r}$, the states $|T \pm, N, M\rangle$ become more strongly coupled to the $\mathrm{CT}$ coherence states $|C \pm, N \mp n, M \pm 1\rangle$, since their zeroth-order energy difference $\Delta \omega^{(0)}$ is approximately zero, thus requiring explicit mixing of these states. As was demonstrated for the $I=3 / 2$ case, this degenerate mixing is the basis for conversions of triplequantum coherence into CT coherence at RR conditions. ${ }^{20}$ Conversion of five-quantum coherence into CT coherence also is favored at RR conditions. Although there exists a secondorder coupling $V_{C \pm, q \pm}^{(2)}$ between the five-quantum and $\mathrm{CT}$ coherences, these coherences can be coupled indirectly through the triple-quantum coherences: the five-quantum coherences 
are coupled directly to the triple-quantum coherences by the first-order coupling $V_{q \pm, T \pm}^{(1)}$, and in turn the triple-quantum coherences are coupled directly to the CT coherences by the first-order coupling $V_{T \pm, C \pm}^{(1)}$. The relative importance of the direct versus indirect couplings for five-quantum to CT conversion is discussed later in the numerical simulations section. Finally, as a result of the mixing of CT coherence with five-quantum and triple-quantum coherences at RR conditions, the excitation efficiencies of both five-quantum and triplequantum coherences have minima at these conditions, as does the efficiency of conversion between five-quantum and triple-quantum coherences.

\section{B. Away from RR conditions}

Away from RR conditions $\left(\omega_{1} \neq \frac{2}{3} n \omega_{r}\right)$ the states $|T \pm, N, M\rangle$ are more weakly coupled to the states $\left|C \pm, N^{\prime}, M^{\prime}\right\rangle$. This enables a stronger mixing between the states $|T \pm, N, M\rangle$ and $|q \pm, N, M\rangle$, and in the problem may be simplified further by considering only the dynamics in the four-dimensional degenerate subspace $\{|T \pm, N, M\rangle,|q \pm, N, M\rangle\}$. The energies may be calculated to second order and the eigenstates to zeroth order in the degenerate subspace by diagonalizing the following matrix:

$$
\widehat{H}_{F}^{N, M}=\left(N \omega_{r}+\frac{3 M}{2} \omega_{1}\right) \widehat{1}+\left(\begin{array}{cccc}
\bar{V}_{q+, q+}^{(2)} & \bar{V}_{q+, T+}^{(1)} & 0 & 0 \\
\bar{V}_{T+, q+}^{(1)} & \bar{V}_{T+, T+}^{(2)} & 0 & 0 \\
0 & 0 & \bar{V}_{T-, T-}^{(2)} & \bar{V}_{T-, q-}^{(1)} \\
0 & 0 & \bar{V}_{q-, T-}^{(1)} & \bar{V}_{q-, q-}^{(2)}
\end{array}\right) .
$$

The explicit diagonalization of $\widehat{H}_{F}^{N, M}$ in Eq. 16 gives some insight into the dynamics away from rotary resonance conditions. Defining the following:

$$
\begin{aligned}
\bar{V}_{q \pm, T \pm}^{(1)} & =\bar{V}_{T \mp, q \mp}^{*(1)}=\frac{\sqrt{5} \omega_{1}\left(P_{0}+i Q_{0}\right)}{2} \\
\bar{V}_{q \pm, q \pm}^{(2)} & =\frac{5 i \omega_{1}^{2}}{2 \omega_{r}} \sum_{N>0}^{\infty} \frac{P_{N} Q_{-N}-Q_{N} P_{-N}}{N} \\
\bar{V}_{T \pm, T \pm}^{(2)} & =-\bar{V}_{q \pm, q \pm}^{(2)}+16 \omega_{1}^{2} \omega_{r} \sum_{N>0}^{\infty} \frac{N i\left[R_{N} S_{-N}-S_{N} R_{-N}\right]}{9 \omega_{1}^{2}-4 N^{2} \omega_{r}^{2}}
\end{aligned}
$$




$$
\begin{aligned}
& \mp \frac{4 \omega_{1}}{3}\left(\left|R_{0}\right|^{2}+\left|S_{0}\right|^{2}\right) \mp 24 \omega_{1}^{3} \sum_{N>0}^{\infty} \frac{\left|R_{N}\right|^{2}+\left|S_{N}\right|^{2}}{9 \omega_{1}^{2}-4 N^{2} \omega_{r}^{2}} \\
\delta= & \frac{\left(\bar{V}_{T+, T+}^{(2)}-\bar{V}_{T-, T-}^{(2)}\right)}{2} \\
\Delta_{-}= & \sqrt{\left|\bar{V}_{T-, q-}^{(1)}\right|^{2}+\left(\frac{\bar{V}_{q-, q-}^{(2)}-\bar{V}_{T-, T-}^{(2)}}{2}\right)^{2}} \\
\Delta_{+}= & \sqrt{\left|\bar{V}_{T+, q+}^{(1)}\right|^{2}+\left(\frac{\bar{V}_{q+, q+}^{(2)}-\bar{V}_{T+, T+}^{(2)}}{2}\right)^{2}} \\
\sin \left(\theta_{+}\right)= & \frac{\bar{V}_{q+, q+}^{(2)}-\bar{V}_{T+T+}^{(2)}-2 \Delta_{+}}{2 \sqrt{\left|\bar{V}_{T+, q+}^{(1)}\right|^{2}+\left(\frac{\bar{V}_{q+, q+}^{(2)}-\bar{V}_{T+, T+}^{(2)}}{2}-\Delta_{+}\right)^{2}}} \\
\cos \left(\theta_{+}\right)= & \frac{\left|\bar{V}_{T+, q+}\right|}{\sqrt{\left|\bar{V}_{T+, q+}^{(1)}\right|^{2}+\left(\frac{\left.\bar{V}_{q+, q+}^{(2)}-\bar{V}_{T+, T+}^{(2)}-\Delta_{+}\right)^{2}}{2}\right.}} \\
\sin \left(\theta_{-}\right)= & \frac{\bar{V}_{q-, q-}^{(2)}-\bar{V}_{T-T-}^{(2)}-2 \Delta_{-}}{2 \sqrt{\left|\bar{V}_{T-, q-}^{(1)}\right|^{2}+\left(\frac{\bar{V}_{q-, q-}^{(2)}-\bar{V}_{T-, T-}^{(2)}-\Delta_{-}}{2}\right)^{2}}} \\
\cos \left(\theta_{-}\right)= & \frac{\left|\bar{V}_{T-, q-}\right|}{\sqrt{\left|\bar{V}_{T-, q-}^{(1)}\right|^{2}+\left(\frac{\left.\bar{q}_{q-, q-}^{(2)}-\bar{V}_{T-, T-}^{(2)}-\Delta_{-}\right)^{2}}{2}\right.}}
\end{aligned}
$$

Consider an initial density matrix equal to $I_{Z}$ but with the CT $z$-magnetization neglected (since CT magnetization does not evolve, in the approximation where the CT states are neglected in $\left.\widehat{H}_{F}^{N, M}\right)$ :

$$
\begin{aligned}
\rho(0)= & \sum_{N, M} \frac{5}{2}[|q+, N, M\rangle\langle q-, N, M|+| q-, N, M\rangle\langle q+, N, M|] \\
& +\frac{3}{2}[|T+, N, M\rangle\langle T-, N, M|+| T-, N, M\rangle\langle T+, N, M|]
\end{aligned}
$$

In the absence of the second-order quadrupolar interaction and resonance offsets, $\rho(0)$ will develop multiple-quantum coherences with phase Y. Taking $\exp (i S) \approx \hat{1}$, the expectation values for both triple-quantum $\left(\left\langle I_{Y}^{2-5}\right\rangle\right)$ and five-quantum $\left(\left\langle I_{Y}^{1-6}\right\rangle\right)$ coherences are

$$
\begin{aligned}
\left\langle I_{Y}^{2-5}\right\rangle(t)= & -5 \sin \left(2 \theta_{+}\right) \sin \left(2 \theta_{-}\right) \sin \left(\Delta_{-} t\right) \sin \left(\Delta_{+} t\right) \sin (\delta t) \\
& -3\left[\sin ^{2}\left(\theta_{+}\right) \sin ^{2}\left(\theta_{-}\right) \sin \left(\left(\Delta_{+}-\Delta_{-}+\delta\right) t\right)\right.
\end{aligned}
$$




$$
\begin{aligned}
& -\cos ^{2}\left(\theta_{+}\right) \cos ^{2}\left(\theta_{-}\right) \sin \left(\left(\Delta_{+}-\Delta_{-}-\delta\right) t\right) \\
& +\sin ^{2}\left(\theta_{+}\right) \cos ^{2}\left(\theta_{-}\right) \sin \left(\left(\Delta_{+}+\Delta_{-}+\delta\right) t\right) \\
& \left.-\cos ^{2}\left(\theta_{+}\right) \sin ^{2}\left(\theta_{-}\right) \sin \left(\left(\Delta_{+}+\Delta_{-}-\delta\right) t\right)\right] \\
\left\langle I_{Y}^{1-6}\right\rangle(t)= & 5\left[\sin ^{2}\left(\theta_{+}\right) \sin ^{2}\left(\theta_{-}\right) \sin \left(\left(\Delta_{+}-\Delta_{-}-\delta\right) t\right)\right. \\
& -\cos ^{2}\left(\theta_{+}\right) \cos ^{2}\left(\theta_{-}\right) \sin \left(\left(\Delta_{+}-\Delta_{-}+\delta\right) t\right) \\
& +\sin ^{2}\left(\theta_{+}\right) \cos ^{2}\left(\theta_{-}\right) \sin \left(\left(\Delta_{+}+\Delta_{-}-\delta\right) t\right) \\
& \left.-\cos ^{2}\left(\theta_{+}\right) \sin ^{2}\left(\theta_{-}\right) \sin \left(\left(\Delta_{+}+\Delta_{-}+\delta\right) t\right)\right] \\
& -3 \sin \left(2 \theta_{+}\right) \sin \left(2 \theta_{-}\right) \sin \left(\Delta_{+} t\right) \sin \left(\Delta_{-} t\right) \sin (\delta t)
\end{aligned}
$$

The triple-quantum and five-quantum excitation efficiencies represented by Eqs. 19 and Eq. 20 are plotted in Figures 3(A) and (B) respectively as a function of $\omega_{1}$ and time for a powdered sample. In the calculations, $\bar{V}_{T \pm, T \pm}^{(2)}$ is replaced with $V_{T \pm, T \pm}^{(2)}$ (as defined in Appendix C) when near the RR conditions $\omega_{1}=\frac{2}{3} n \omega_{r}$, due to the singularity in the denominator of $\bar{V}_{T \pm, T \pm}^{(2)}$. Note that when $\delta$ (which roughly represents the energy difference between the states $|T+, N, M\rangle$ and $|T-, N, M\rangle)$ is close to zero, not only does $\left\langle I_{Y}^{2-5}\right\rangle(t) \approx 0$ for all times, but also $\left\langle I_{Y}^{1-6}\right\rangle(t) \approx 0$. The terms in the expectation values proportional to $\sin (\delta t)$ oscillate between positive and negative values, with zeroes at roughly $\omega_{1}=\frac{1}{3} n \omega_{r}$, as shown in Figures 3(A) and (B). Such behavior was observed previously in the spin $I=3 / 2$ case and was a result of a destructive interference between the various crystallite orientations in a powdered sample. ${ }^{20}$ Additional zeroes occur due to the other terms in Eqs. 19 and 20 when $\omega_{1}$ is in between $\frac{1}{3} n \omega_{r}$ and $\frac{2}{3} n \omega_{r}$; these zeroes are especially pronounced in the case of the five-quantum excitation.

Additionally, conversions between five-quantum and triple-quantum coherences, i.e. $+5 \mathrm{Q} \rightarrow+3 \mathrm{Q}$ and $+5 \mathrm{Q} \rightarrow-3 \mathrm{Q}$, are both possible. Starting with initial $+5 \mathrm{Q}$ coherence $(\rho(0)=|5 / 2\rangle\langle-5 / 2|)$ the expectation values for the conversions are

$$
\begin{aligned}
\langle \pm 3 Q(t)\rangle= & \frac{1}{2}\left[\sin ^{2}\left(\theta_{+}\right) \cos ^{2}\left(\theta_{+}\right)\left(1-\cos \left(2 \Delta_{+} t\right)\right)+\sin ^{2}\left(\theta_{-}\right) \cos ^{2}\left(\theta_{-}\right)\left(1-\cos \left(2 \Delta_{-} t\right)\right)\right. \\
& \left. \pm \sin \left(2 \theta_{+}\right) \sin \left(2 \theta_{-}\right) \sin \left(\Delta_{+} t\right) \sin \left(\Delta_{-} t\right) \cos (\delta t)\right]
\end{aligned}
$$


It can be seen from this equation that when $\delta$ is small, the $+5 \mathrm{Q} \rightarrow+3 \mathrm{Q}$ pathway is the most efficient, and the $+5 \mathrm{Q} \rightarrow-3 \mathrm{Q}$ pathway is suppressed. Thus, maxima for $+5 \mathrm{Q} \rightarrow+3 \mathrm{Q}$ are expected at the conditions $\omega_{1}=\frac{1}{3} n \omega_{r}$, where $n$ is an odd integer. The $+5 \mathrm{Q} \rightarrow+3 \mathrm{Q}$ conversion efficiency represented by Eq. 21 is plotted in Fig. 3(C) and shows that maxima in the conversion efficiency occur in between the RR conditions.

\section{Second-order quadrupolar effects, resonance offsets and CSA}

So far the treatment has neglected the effects of the second-order quadrupolar interaction, resonance offsets, and chemical shift anisotropy. These effects can be included by adding the term $H^{\prime}(t)$ to the Hamiltonian in Eq. 1, where

$$
\begin{aligned}
H^{\prime}(t)= & H_{Q}^{(2)}(t)+H_{\text {offset }}+H_{C S A}(t) \\
= & \sum_{N=-4}^{4} A_{N} \exp \left[-i N \omega_{r} t\right](|q+\rangle\langle q-|+| q-\rangle\langle q+|) \\
& +B_{N} \exp \left[-i N \omega_{r} t\right](|T+\rangle\langle T-|+| T-\rangle\langle T+|) \\
& +C_{N} \exp \left[-i N \omega_{r} t\right](|C+\rangle\langle C-|+| C-\rangle\langle C+|) .
\end{aligned}
$$

The various contributions of $H_{Q}^{(2)}(t), H_{\text {offset }}$, and $H_{C S A}(t)$ to the coefficents $A_{N}, B_{N}$, and $C_{N}$, can be found in the literature ${ }^{34,35}$. For the case when the CT RF Hamiltonian, $3 \omega_{1} I_{X}^{3-4}$, is less than or comparable in magnitude to the 3-4 (CT) subspace component of $H^{\prime}(t)$, an interaction transformation defined by

$$
V(t)=T\left\{\exp \left(-i \int_{0}^{t} d t^{\prime}\left[H_{Q}^{(1)}\left(t^{\prime}\right)+3 \omega_{1} I_{X}^{3-4}+C_{0} I_{Z}^{3-4}\right]\right)\right\}
$$

is more appropriate than the one used in Eq. 4. In this case, the effective field in the 3-4 subspace lies in the $\mathrm{X}-\mathrm{Z}$ plane, with variable magnitude and direction. These variations cause a broadening of the RR conditions, since each crystallite will experience a different

effective field, leading to different matching conditions, $\omega_{1}^{E F F}=\sqrt{\left(3 \omega_{1}\right)^{2}+C_{0}^{2}}=\frac{2}{3} n \omega_{r}$. In addition, dephasing of the multiple-quantum coherences will occur under the second-order 
quadrupolar coupling, leading to a decrease in the efficiency of multiple-quantum coherence excitation and transfer.

For the case where the RF field is stronger than the magnitude of the $3-4$ subspace component of $H^{\prime}(t)$, the original interaction frame defined by Eq. 4 can be used to obtain

$$
\begin{aligned}
H_{I N T}^{\prime}(t)= & W^{\dagger}(t) H^{\prime}(t) W(t) \\
& \sum_{N=-4}^{4} A_{N} \exp \left[-i N \omega_{r} t\right](|q+\rangle\langle q-|+| q-\rangle\langle q+|) \\
& +B_{N} \exp \left[-i N \omega_{r} t\right](|T+\rangle\langle T-|+| T-\rangle\langle T+|) \\
& +C_{N} \exp \left[-i N \omega_{r} t\right]\left(\exp \left(i 3 \omega_{1} t\right)|C+\rangle\left\langle C-\left|+\exp \left(-i 3 \omega_{1} t\right)\right| C-\right\rangle\langle C+|\right)
\end{aligned}
$$

Transforming into Floquet space adds the term $H_{F}^{\prime}$ to Eq. 8, where

$$
\begin{aligned}
H_{F}^{\prime}= & A_{0}(|q+\rangle\langle q-|+| q-\rangle\langle q+|)+B_{0}(|T+\rangle\langle T-|+| T-\rangle\langle T+|) \\
& +C_{0}\left(b^{2}|C+\rangle\left\langle C-\left|+\left(b^{\dagger}\right)^{2}\right| C-\right\rangle\langle C+|\right)+\sum_{N=1}^{2}\left\{\left(A_{-N} a^{N}+A_{N}\left(a^{\dagger}\right)^{N}\right)\right. \\
& \times(|q+\rangle\langle q-|+| q-\rangle\langle q+|)+\left(B_{-N} a^{N}+B_{N}\left(a^{\dagger}\right)^{N}\right)(|T+\rangle\langle T-|+| T-\rangle\langle T+|) \\
& +\left(C_{-N} a^{N}+C_{N}\left(a^{\dagger}\right)^{N}\right)\left(b^{2}|C+\rangle\left\langle C-\left|+\left(b^{\dagger}\right)^{2}\right| C-\right\rangle\langle C+|\right) .
\end{aligned}
$$

$H_{F}^{\prime}$

con-

nects the subspaces $D_{S+}^{N, M}$ and $D_{S-}^{N, M}$ via the coupling of $|q+, N, M\rangle$ and $|T+, N, M\rangle$ with $|q-, N, M\rangle$ and $|T-, N, M\rangle$, respectively. In addition, near the conditions $\omega_{1}=\frac{1}{3} n \omega_{r}$, the states $|C+, N, M\rangle$ and $|C-, N-n, M+2\rangle$ are degenerate and are directly coupled to each other by $H_{F}^{\prime}$ for $n=1,2,3,4$. This helps to explain the decrease in efficiency for the spinlocking of the CT coherence in between RR conditions that was reported earlier. ${ }^{17}$ Although these additional terms complicate the theoretical treatment, in the past exact numerical calculations on the $I=3 / 2$ system have shown that the basic features of RR remain even in their presence. ${ }^{19,20}$ This will be verified for the $I=5 / 2$ system in the next section. 


\section{NUMERICAL SIMULATIONS}

Although the effective Hamiltonian in Eq. 14 can be diagonalized analytically, the exact solutions are rather complicated and from them little insight is obtained. In order to test how well the effective Hamiltonian (Eq. 14) describes the spin dynamics, exact numerical calculations were conducted and compared to the effective Hamiltonian treatment for a variety of multiple-quantum conversions and excitations. The coefficients in Eq. 6 were calculated for 2000 crystallite orientations generated using REPULSION sampling ${ }^{36}$. In addition, $\exp (i \widehat{S})$ was approximated as $\exp (i \widehat{S}) \approx \widehat{1}+i \lambda \widehat{S}^{(1)}+O\left(\lambda^{2}\right)$, and therefore

$$
\begin{aligned}
\exp \left(-i t H_{F}\right) & =\exp (-i \widehat{S}) \exp \left(-i t \widehat{H}_{F}\right) \exp (i \widehat{S}) \\
& \approx \exp \left(-i \widehat{H}_{F}\right)-i \lambda\left[\widehat{S}^{(1)}, \exp \left(-i t \widehat{H}_{F}\right)\right]
\end{aligned}
$$

This transformation is unitary only up to second-order in $\lambda$, which for high RF powers leads to errors in the calculation of the intensities of the coherences created by multiple-quantum excitations/conversions. In Figs. 4-6, exact numerical simulations are compared with evolution under the effective Hamiltonian in Eq. 14 for the cases of multiple-quantum to CT coherence conversion, multiple-quantum coherence excitation from $z$-magnetization, and five-quantum to triple-quantum coherence conversion, respectively. Maxima in the multiplequantum conversion efficiencies occur at RR conditions (Fig. 4), as predicted in the Theory section. Resonant enhancement of the $+3 \mathrm{Q} \rightarrow+1 \mathrm{Q}(\mathrm{CT})$ coherence conversion (Figs. 4 (A), (B)) is seen only for the first RR condition; this is most likely due to the stronger coupling of the $|T \pm, N, M\rangle$ states to the CT states for large $\omega_{1}$, thus causing a breakdown in the perturbative treatment and a "smoothing" of the $\mathrm{RR}$ conditions. For $+5 \mathrm{Q} \rightarrow+1 \mathrm{Q}$ coherence conversion (Figs. 4 (C), (D)), enhancement of the efficiency is observed at the first two RR conditions. In order to test whether the transfer of $5 \mathrm{Q}$ to CT coherence in the effective Hamiltonian treatment is due mainly to the direct second-order coupling or to the indirect first-order coupling via the triple-quantum coherence states, calculations were performed with the second-order couplings set to zero. The resulting profiles showed 
little change, indicating that the transfer of five-quantum to CT coherence occurs indirectly due to a mixing of the five-quantum coherence states $(|q \pm, N, M\rangle)$ with the triple-quantum coherence states $(|T \pm, N, M\rangle)$, which in turn mix with the CT coherence states $(\mid C \pm, N \mp$ $n, M \pm 1\rangle)$. In Fig. 5, both the effective Hamiltonian treatment [(A), (C)] and the exact numerical calculations demonstrate that the maxima in the multiple-quantum excitation efficiencies occur between RR conditions, with additional minima also occurring in between RR conditions. The reduced effective Hamiltonian treatment presented in the Theory section (Figs. $3(\mathrm{~A}),(\mathrm{B}))$ agrees quite well with the exact numerical simulations. Finally, Fig. 6 indicates that both the effective Hamiltonian treatment and the exact numerical calculations agree for $[(\mathrm{A}),(\mathrm{B})]+5 \mathrm{Q} \rightarrow-3 \mathrm{Q}$ coherence and $[(\mathrm{C}),(\mathrm{D})]+5 \mathrm{Q} \rightarrow+3 \mathrm{Q}$ coherence conversion. The maxima of the $+5 \mathrm{Q} \rightarrow+3 \mathrm{Q}$ coherence conversion efficiency occur roughly in between RR conditions, as was predicted and calculated earlier in the Theory section (Fig. 3 (C)). Additionally, maxima of the $+5 \mathrm{Q} \rightarrow-3 \mathrm{Q}$ coherence conversion efficiency occur in between RR conditions when $\frac{1}{3} n \omega_{r}<\omega_{1}<\frac{2}{3} n \omega_{r}$, and roughly correspond to the dips in the $+5 \mathrm{Q}$ excitation efficiency.

Single crystallite studies were conducted in order to evaluate how well the theory describes the dynamics of individual spins as opposed than powder-averaged results. Three crystallite orientations corresponding to $\beta=10^{\circ}, 45^{\circ}$, and $90^{\circ}$ were chosen due to their varying dependences of $\omega_{Q}(t)$ on $\omega_{r}$. In Fig. 7, exact numerical calculations are compared to the effective Hamiltonian theory for the conversion of $+5 \mathrm{Q}$ coherence into $+3 \mathrm{Q}$ coherence in single crystallites. The effective Hamiltonian approach gives results for $\beta=45^{\circ}$ and $90^{\circ}$ that are quantitatively very close to the exact numerical simulations, whereas the results for $\beta=10^{\circ}$ are less quantitative in nature, although qualitatively correct.

The numerical simulations were performed by directly calculating the amount of coherence created from various initial density matrices (representing either multiple-quantum coherence or $z$-magnetization) while evolving under the system Hamiltonian during an RF pulse. The experiments, to which the simulations are compared, were multiple-pulse MQ- 
MAS experiments. For example, the experimental study of $+5 \mathrm{Q} \rightarrow+3 \mathrm{Q}$ coherence conversion under RR conditions utilized a "hard" RF pulse to create $+5 \mathrm{Q}$ coherence, a soft "RR" $+5 \mathrm{Q} \rightarrow+3 \mathrm{Q}$ coherence conversion pulse, a hard $+3 \mathrm{Q} \rightarrow+1 \mathrm{Q}$ coherence conversion pulse, and a soft selective inversion pulse to create detectable $-1 \mathrm{Q}$ coherence. In this case, the measure of the $+5 \mathrm{Q} \rightarrow+3 \mathrm{Q}$ conversion efficiency was the magnitude of the MQMAS signal as a function of the RR conversion pulse strength and duration.

\section{EXPERIMENTAL PARAMETERS}

The experiments were performed using a Chemagnetics/Varian CMX Infinity 500 spectrometer equipped with a $3.2 \mathrm{~mm}$ Chemagnetics MAS probe. A $20 \mathrm{kHz}$ spinning speed

was used for all experiments. The ${ }^{27} \mathrm{Al}$ NMR spectra were obtained at Larmor frequency of 130.3 MHz. Multiple-quantum coherences were generated using a $150 \mathrm{kHz}$ pulse of duration $2.3 \mu$ s for $+3 \mathrm{Q}$ creation and $3.4 \mu$ s for $+5 \mathrm{Q}$ creation, and a $150 \mathrm{kHz} 0.45 \mu$ s pulse was used for $+3 \mathrm{Q}$ to $+1 \mathrm{Q}$ coherence conversion in the $+5 \mathrm{Q}$ to $+3 \mathrm{Q}$ coherence $\mathrm{RR}$ conversion experiment. The split- $t_{1}$ version of the MQMAS experiment was used. ${ }^{37}$ Only 1D MQMAS experiments were performed, with a fixed $t_{1}$ interval of $2.8 \mu$ s to separate the pulses. Experimental profiles of the $\mathrm{RR}$ effects in $+5 \mathrm{Q} \rightarrow+3 \mathrm{Q}$ coherence and $+3 \mathrm{Q} \rightarrow+1 \mathrm{Q}$ coherence conversion pulses were obtained by varying the amplitude of the corresponding pulse in the split- $t_{1}$ experiment. The phase cycles employed for the $+3 \mathrm{Q} \rightarrow+1 \mathrm{Q}$ and $+5 \mathrm{Q} \rightarrow+3 \mathrm{Q}$ coherence conversion pulses are given in Table I. The sample of aluminum acetylacetonate (Aldrich, 99+\%, used without further purification) was ground using a mortar and pestle before it was packed into a zirconia rotor. The quadrupolar parameters for this sample have been reported to be $C_{Q}=3.0 \mathrm{MHz}$ and $\eta=0.15 .^{38}$ Calibration of the RF amplitudes was performed using a $1 \mathrm{M} \mathrm{AlCl}_{3}(\mathrm{aq})$ sample. 


\section{RESULTS AND DISCUSSION}

Fig. 8 gives the experimental profiles for $(\mathrm{A})+5 \mathrm{Q} \rightarrow+3 \mathrm{Q}$ coherence conversion and (B) $+3 \mathrm{Q} \rightarrow+1 \mathrm{Q}(\mathrm{CT})$ coherence conversion, respectively. The experimental profiles show qualitative agreement with the respective theoretical and numerical results in Fig. 5 and Fig. 6. There is a maximum of triple-quantum conversion efficiency at the first RR condition; at RR conditions, the triple-quantum states are strongly coupled to the CT states. As $\omega_{1}$ increases, the triple-quantum states become more strongly coupled to the CT states, thus "smoothing" out the RR conditions. This was observed experimentally (Fig. 8(B)).

The $+5 \mathrm{Q} \rightarrow+3 \mathrm{Q}$ coherence conversion shows maxima in between $\mathrm{RR}$ conditions, as predicted in the Theory section. Although the exact numerical calculation and the effective Hamiltonian theory both predict that the most efficient conversion occurs around $\frac{\omega_{1}}{2 \pi}=6.5$ $\mathrm{kHz}$, the experimental results give a slightly larger intensity at $\frac{\omega_{1}}{2 \pi} \approx 20 \mathrm{kHz}$ (Fig. 8). This discrepency is a result of the second-order quadrupolar coupling, as shown by exact numerical calculation in Fig. 9. Using larger RF power helps to lessen the effects of the second-order quadrupolar coupling and resonance offsets, making the coherence transfer more efficient, as explained earlier in the Theory section. The design of low power sequences that are robust to offsets, CSA, and second-order quadrupolar coupling therefore would help to improve the efficiencies of these techniques.

In conventional MQMAS experiments for $I=5 / 2$ quadrupolar nuclei, evolution of either the $+5 \mathrm{Q}$ or $+3 \mathrm{Q}$ coherence followed by evolution of the CT coherence is used to cancel the remaining (after MAS) anisotropic second-order quadrupolar interaction. It was shown recently that evolution of the $+5 \mathrm{Q}$ coherence followed by evolution of the $+3 \mathrm{Q}$ coherence can in some cases improve the resolution of the MQMAS by increasing the scaling factor for the chemical shift and isotropic quadrupolar interactions, a technique called mixed multiplequantum MAS (MMQMAS). ${ }^{39,40}$ The efficiency of the $+5 \mathrm{Q} \rightarrow+3 \mathrm{Q}$ coherence transfer can be greatly improved using the low power conversion pulses in between RR conditions, enabling greater sensitivity for the MMQMAS experiments. Such work will be presented elsewhere. 


\section{CONCLUSIONS}

The theoretical formalism we developed for the spin dynamics of $I=3 / 2$ quadrupolar nuclei in the sudden-passage $\operatorname{limit}^{20}$ has been applied successfully to the $I=5 / 2$ case. Transforming into the Quadrupolar/Central-Transition frame clearly shows the conditions at which mixing between the various spin states occurs, thus enabling the prediction of the various multiple-quantum coherence conversions and excitations that occur under conditions of low RF power and fast MAS (i.e., the sudden-passage limit). Bimodal Floquet theory was utilized in this frame, and the spin dynamics could be understood by examining the effective Hamiltonian in two three-dimensional subspaces (Fig. 1). When $\omega_{1}=\frac{2}{3} n \omega_{r}$ ( $n$ integral), the triple-quantum Floquet states $|T \pm, N, M\rangle$ and the CT Floquet states $|C \pm, N \mp n, M \pm 1\rangle$ are strongly mixed. This results in coherence transfer between the triple-quantum and CT coherences, and thus in poor spin-locking efficiency of the CT coherences. Transfer of five-quantum to CT coherence also is possible, mostly due to the fact that the fivequantum Floquet states $|q \pm, N, M\rangle$ are directly coupled to the triple-quantum Floquet states $|T \pm, N, M\rangle$. In between RR conditions, the triple quantum and CT Floquet states are only weakly coupled, and thus efficient spin locking is possible. This also enables the coherence transfer between five-quantum and triple-quantum coherences to become more effective, by suppressing the competing triple-quantum to CT coherence pathway. For multiple-quantum excitations from equilibrium $z$-magnetization, maxima in the efficiencies occur in between RR conditions, with minimum efficiency at the RR conditions. Additional minima also occur almost halfway between the RR conditions. These additional minima, similar to those observed in $I=3 / 2$ systems, are a result of the powder averaging over crystallite orientations, whereas the minima at the RR conditions are due to direct coupling between

the multiple-quantum and CT coherences. Excitation of five-quantum coherence is a result of the coupling between the five-quantum $|q \pm, N, M\rangle$ and triple-quantum $|T \pm, N, M\rangle$ states. For $+5 \mathrm{Q} \rightarrow+3 \mathrm{Q}$ coherence conversion, maxima in the efficiency occur roughly halfway between RR conditions; this is a result of the weak coupling between the triple-quantum 
and CT coherences, along with a near-degeneracy of the $|T \pm, N, M\rangle$ states. Our theoretical model was tested against exact numerical simulations and compared to experiment, and the theoretical predictions were found to agree with both.

An understanding of the dynamics of quadrupolar nuclei is useful in the design of experiments that optimize the signal produced from various coherence pathways. We are currently using our theoretical framework to examine the possibility of using "slow" amplitude and phase modulation in order to enhance multiple-quantum coherence excitations and conversions beyond those obtained by simple spin-locking. We also are developing sequences that are more robust to the second-order quadrupolar interaction.

\section{ACKNOWLEDGMENTS}

This work was supported by the Director, Office of Energy Research, Office of Basic Energy Sciences, Materials Sciences Division, U.S. Department of Energy under Contract No. DE-AC03-76SF00098. We would like to thank Z. H. Gan et. al for providing us with a

preprint of their paper. ${ }^{24}$ We would also like to thank Dr. S. Wi for bringing to our attention the reference of M. Baldus et al. ${ }^{18}$

\section{Appendix A}

The coefficients introduced in Eq. 2 are given by:

$$
\begin{aligned}
C_{1} & =-\frac{3}{8} \sin (2 \theta) \sin (2 \beta)\left[1-\frac{\eta}{3} \cos (2 \alpha)\right] \\
C_{2} & =\frac{3}{8} \sin ^{2}(\theta)\left[\sin ^{2}(\beta)+\frac{\eta}{3}\left(\cos ^{2}(\beta)+1\right) \cos (2 \alpha)\right] \\
S_{1} & =-\frac{\eta}{4} \sin (2 \theta) \sin (\beta) \sin (2 \alpha) \\
S_{2} & =\frac{\eta}{4} \sin ^{2}(\theta) \cos (\beta) \sin (2 \alpha) \\
\omega_{Q} & =2 \pi \frac{C_{Q}}{2 I(2 I-1)}
\end{aligned}
$$


where $C_{Q}=\frac{e^{2} q Q}{h}$ is the quadrupolar coupling constant and $\eta$ is the quadrupolar asymmetry parameter. The Euler angles $(\alpha, \beta, \gamma)$ relate the quadrupolar PAS to the rotor-fixed coordinate system, and $\theta$ relates the rotor axis to the Zeeman field. For the case of MAS, $\theta=\arctan (\sqrt{2})$.

\section{Appendix B}

As was shown in an earlier work for the case of $I=3 / 2$ nuclei, a transformation into bimodal Floquet space can give quantitative insight into the spin dynamics in the sudden passage limit. ${ }^{20}$ However, the same results also can be obtained by using unimodal Floquet theory, where the interaction frame is defined only by the first-order quadrupolar interaction. In this quadrupolar interaction frame the $I=3 / 2$ Hamiltonian under MAS and spin locking is given by

$$
\begin{aligned}
H_{I N T}= & 2 \omega_{1} I_{X}^{2-3}+\sqrt{3} \omega_{1}\left(I_{X}^{1-2}+I_{X}^{3-4}\right) \cos \left(\int_{0}^{t} d t^{\prime} \omega_{Q}\left(t^{\prime}\right)\right)+\left(I_{Y}^{3-4}-I_{Y}^{1-2}\right) \sin \left(\int_{0}^{t} d t^{\prime} \omega_{Q}\left(t^{\prime}\right)\right) \\
= & 2 \omega_{1} I_{X}^{2-3} \\
& +\sqrt{3} \omega_{1} \sum_{N=-\infty}^{\infty} A_{N} \exp \left(-i N \omega_{r} t\right)\left(I_{X}^{1-2}+I_{X}^{3-4}\right)+B_{N} \exp \left(-i N \omega_{r} t\right)\left(I_{Y}^{3-4}-I_{Y}^{1-2}\right)
\end{aligned}
$$

The time dependence can be removed by transforming into Floquet space, giving the unimodal Floquet Hamiltonian

$$
\begin{aligned}
H_{F}^{U} & =N^{r} \omega_{r}+\omega_{1}|C+\rangle\left\langle C+\left|-\omega_{1}\right| C-\right\rangle\langle C-|+\frac{\sqrt{3}}{2} \omega_{1}\left[\left(A_{0}+i B_{0}\right)(|T+\rangle\langle C+|+| T-\rangle\langle C-|)\right. \\
& \left.+\left(A_{0}-i B_{0}\right)(|C+\rangle\langle T+|+| C-\rangle\langle T-|)\right]+\sum_{N=1}^{\infty}\left(a^{\dagger}\right)^{N}\left[\left(A_{N}+i B_{N}\right)(|T+\rangle\langle C+|+| T-\rangle\langle C-|)\right. \\
& \left.+\left(A_{N}-i B_{N}\right)(|C+\rangle\langle T+|+| C-\rangle\langle T-|)\right]+a^{N}\left[\left(A_{-N}-i B_{-N}\right)(|C+\rangle\langle T+|+| C-\rangle\langle T-|)\right. \\
& \left.+\left(A_{-N}+i B_{-N}\right)(|T+\rangle\langle C+|+| T-\rangle\langle C-|)\right]
\end{aligned}
$$

As in the $I=5 / 2$ case, an effective Hamiltonian can be constructed in order to help simplify the analysis of $H_{F}^{U}$. The subspaces $D_{S \pm}^{N}=\{|T \pm, N\rangle,|C \pm, N \mp n\rangle\}$ are constructed, where 
$n$ is a positive integer that minimizes

$$
\frac{\left|n \omega_{r}-\omega_{1}\right|}{\sqrt{\left(n \omega_{r}-\omega_{1}\right)^{2}+\frac{3}{4} \omega_{1}^{2}\left|A_{n}+i B_{n}\right|^{2}}}
$$

which is analogous to Eq. 11 for the $I=5 / 2$ case. The effective Hamiltonian in each $D_{S \pm}^{N}$ subspace is given by

$$
\widehat{H}_{F}^{N}=N \omega_{r} \widehat{1}+\left(\begin{array}{cccc}
V_{T+, T+}^{(2)} & V_{T+, C+}^{(1)} & 0 & 0 \\
V_{C+, T+}^{(1)} & V_{C+, C+}^{(2)}+\Delta \omega^{(0)} & 0 & 0 \\
0 & 0 & V_{C-, C-}^{(2)}-\Delta \omega^{(0)} & V_{C-, T-}^{(1)} \\
0 & 0 & V_{T-, C-}^{(1)} & V_{T-, T-}^{(2)}
\end{array}\right)
$$

where $\Delta \omega^{(0)}=\omega_{1}-n \omega_{r}$ and

$$
\begin{aligned}
& V_{T+, T+}^{(2)}=-\frac{3}{4} \omega_{1}^{2} \sum_{N \neq-n} \frac{\left(A_{N}-i B_{N}\right)\left(A_{-N}+i B_{-N}\right)}{\omega_{1}+N \omega_{r}} \\
& V_{C+, C+}^{(2)}=\frac{3}{4} \omega_{1}^{2} \sum_{N \neq n} \frac{\left(A_{N}+i B_{N}\right)\left(A_{-N}-i B_{-N}\right)}{\omega_{1}-N \omega_{r}} \\
& V_{C-, C-}^{(2)}=-\frac{3}{4} \omega_{1}^{2} \sum_{N \neq-n} \frac{\left(A_{N}+i B_{N}\right)\left(A_{-N}-i B_{-N}\right)}{\omega_{1}+N \omega_{r}} \\
& V_{T-, T-}^{(2)}=\frac{3}{4} \omega_{1}^{2} \sum_{N \neq n} \frac{\left(A_{N}-i B_{N}\right)\left(A_{-N}+i B_{-N}\right)}{\omega_{1}-N \omega_{r}} \\
& V_{T+, C+}^{(1)}=V_{C+, T+}^{*(1)}=\frac{\sqrt{3} \omega_{1}}{2}\left(A_{n}+i B_{n}\right) \\
& V_{T-, C-}^{(1)}=V_{C-, T-}^{*(1)}=\frac{\sqrt{3} \omega_{1}}{2}\left(A_{-n}+i B_{-n}\right)
\end{aligned}
$$

In the bimodal Floquet treatment, the $I=3 / 2$ Floquet Hamiltonian is given by

$$
\begin{aligned}
H_{F}^{B}= & \omega_{r} N^{r}+\omega_{1} N^{1}+\frac{\sqrt{3} \omega_{1}}{2}\left\{b^{\dagger}\left[\left(A_{0}+i B_{0}\right)|T-\rangle\left\langle C-\left|+\left(A_{0}-i B_{0}\right)\right| C+\right\rangle\langle T+|\right]\right. \\
& +b\left[\left(A_{0}+i B_{0}\right)|T+\rangle\left\langle C+\left|+\left(A_{0}-i B_{0}\right)\right| C-\right\rangle\langle T-|\right] \\
& +\frac{\sqrt{3} \omega_{1}}{2} \sum_{N=1}^{\infty}\left(a^{\dagger}\right)^{N} b^{\dagger}\left[\left(A_{N}+i B_{N}\right)|T-\rangle\left\langle C-\left|+\left(A_{N}-i B_{N}\right)\right| C+\right\rangle\langle T+|\right] \\
& +a^{N} b^{\dagger}\left[\left(A_{-N}+i B_{-N}\right)|T-\rangle\left\langle C-\left|+\left(A_{-N}-i B_{-N}\right)\right| C+\right\rangle\langle T+|\right] \\
& +\left(a^{\dagger}\right)^{N} b\left[\left(A_{N}+i B_{N}\right)|T+\rangle\left\langle C+\left|+\left(A_{N}-i B_{N}\right)\right| C-\right\rangle\langle T-|\right] \\
& +a^{N} b\left[\left(A_{-N}+i B_{-N}\right)|T+\rangle\left\langle C+\left|+\left(A_{-N}-i B_{-N}\right)\right| C-\right\rangle\langle T-|\right],
\end{aligned}
$$


where $b, b^{\dagger}, a, a^{\dagger}$ act similarly to the raising and lowering operators defined in Eq. 9. An effective Hamiltonian can be constructed in order to simplify the analysis of $H_{F}^{B}$. The relevant subspaces are given by $D_{S \pm}^{N, M}=\{|T \pm, N, M\rangle,|C \pm, N \mp n, M \pm 1\rangle\}$, where $n$ is chosen using Eq. 30. An effective Hamiltonian, $\widehat{H}_{F}^{N, M}$, is given to second-order as

$$
\widehat{H}_{F}^{N, M}=\left(N \omega_{r}+M \omega_{1}\right) \widehat{1}+\left(\begin{array}{cccc}
V_{T+, T+}^{(2)} & V_{T+, C+}^{(1)} & 0 & 0 \\
V_{C+, T+}^{(1)} & V_{C+, C+}^{(2)}+\Delta \omega^{(0)} & 0 & 0 \\
0 & 0 & V_{C-, C-}^{(2)}-\Delta \omega^{(0)} & V_{C-, T-}^{(1)} \\
0 & 0 & V_{T-, C-}^{(1)} & V_{T-, T-}^{(2)}
\end{array}\right)
$$

where $V_{i, j}^{(2)}$ and $V_{i, j}^{(1)}$ are given in Eq. 32. The effective Hamiltonians in Eq. 31 and Eq. 34 differ only by a constant energy term, and there exists a one-to-one mapping, $|T \pm, N\rangle \leftrightarrow$ $|T \pm, N, M\rangle$ and $|C \pm, N \mp n\rangle \leftrightarrow|C \pm, N \mp n, M \pm 1\rangle$. Therefore, it can be seen that the bimodal Floquet treatment is just a convenient way to keep track of the energy differences between the $|T \pm\rangle$ states and the $|C \pm\rangle$ states, as shown in Fig. 10.

\section{Appendix C}

Neglecting second-order quadrupolar coupling and resonance offset terms, the coefficients (Eq. 15) of the effective Hamiltonian $\widehat{H}_{F}^{N, M}$ of Eq. 14 are given explicitly by

$$
\begin{aligned}
& V_{q+, q+}^{(2)}=V_{q-, q-}^{(2)}=\frac{5 i \omega_{1}^{2}}{2 \omega_{r}} \sum_{N>0}^{\infty} \frac{P_{N} Q_{-N}-Q_{N} P_{-N}}{N} \\
& V_{q+, T+}^{(1)}=V_{T+, q+}^{*(1)}=V_{q-, T-}^{(1)}=V_{T-, q-}^{*(1)}=\frac{\sqrt{5} \omega_{1}\left(P_{0}+i Q_{0}\right)}{2} \\
& V_{T+, T+}^{(2)}=-\frac{5 i \omega_{1}^{2}}{2 \omega_{r}} \sum_{N>0}^{\infty} \frac{P_{N} Q_{-N}-Q_{N} P_{-N}}{N}-2 \omega_{1}^{2} \sum_{N \neq-n} \frac{\left(R_{N}-i S_{N}\right)\left(R_{-N}+i S_{-N}\right)}{\frac{3}{2} \omega_{1}+N \omega_{r}} \\
& V_{T-, T-}^{(2)}=-\frac{5 i \omega_{1}^{2}}{2 \omega_{r}} \sum_{N>0}^{\infty} \frac{P_{N} Q_{-N}-Q_{N} P_{-N}}{N}+2 \omega_{1}^{2} \sum_{N \neq n} \frac{\left(R_{N}-i S_{N}\right)\left(R_{-N}+i S_{-N}\right)}{\frac{3}{2} \omega_{1}-N \omega_{r}} \\
& V_{T+, C+}^{(1)}=V_{C+, T+}^{*(1)}=\sqrt{2} \omega_{1}\left(R_{n}+i S_{n}\right) \\
& V_{T-, C-}^{(1)}=V_{C-, T-}^{*(1)}=\sqrt{2} \omega_{1}\left(R_{-n}+i S_{-n}\right) \\
& V_{q+, C+}^{(2)}=V_{C+, q+}^{*(2)}=\frac{\sqrt{10} \omega_{1}^{2}}{4} \sum_{N \neq 0}\left(R_{(n+N)}+i S_{(n+N)}\right)\left(P_{-N}+i Q_{-N}\right)\left[\frac{-1}{N \omega_{r}}+\frac{1}{\frac{3}{2} \omega_{1}-(N+n) \omega_{r}}\right]
\end{aligned}
$$




$$
\begin{aligned}
V_{q-, C-}^{(2)} & =V_{C-, q-}^{*(2)}=\frac{\sqrt{10} \omega_{1}^{2}}{4} \sum_{N \neq 0}\left(R_{N-n}+i S_{N-n}\right)\left(P_{-N}+i Q_{-N}\right)\left[\frac{-1}{N \omega_{r}}+\frac{1}{(N-n) \omega_{r}-\frac{3}{2} \omega_{1}}\right] \\
V_{C+, C+}^{(2)} & =2 \omega_{1}^{2} \sum_{N \neq n} \frac{\left(R_{N}+i S_{N}\right)\left(R_{-N}-i S_{-N}\right)}{\frac{3}{2} \omega_{1}-N \omega_{r}} \\
V_{C-, C-}^{(2)} & =-2 \omega_{1}^{2} \sum_{N \neq-n} \frac{\left(R_{N}+i S_{N}\right)\left(R_{-N}-i S_{-N}\right)}{\frac{3}{2} \omega_{1}+N \omega_{r}}
\end{aligned}
$$

Diagonalization of Eq. 14 in the subspaces $D_{S \pm}^{N, M}$ gives the eigenvalues to second order in $\lambda$ and the eigenvectors to zeroth order.

\section{Appendix D}

Consider the case of a crystallite with the Euler angles $(\alpha, \beta, \gamma)=\left(0^{\circ}, 90^{\circ}, 0^{\circ}\right)$ relating its quadrupolar PAS to the Zeeman field axis. From Appendix A, $\omega_{Q}(t)=\frac{\omega_{Q}}{4} \cos \left(2 \omega_{r} t\right)$, and so

$$
\int_{0}^{t} \omega_{Q}\left(t^{\prime}\right) d t^{\prime}=\frac{\omega_{Q}}{8 \omega_{r}} \sin \left(2 \omega_{r} t\right)
$$

Therefore, the coefficients in Eq. 6 can be written in terms of spherical Bessel functions as

$$
\begin{aligned}
\cos \left(12 \int_{0}^{t} \omega_{Q}\left(t^{\prime}\right) d t^{\prime}\right) & =\sum_{N=-\infty}^{\infty} J_{2 N}\left(\frac{3 \omega_{Q}}{2 \omega_{r}}\right) \exp \left(-i 4 N \omega_{r} t\right) \\
\sin \left(12 \int_{0}^{t} \omega_{Q}\left(t^{\prime}\right) d t^{\prime}\right) & =-i \sum_{N=-\infty}^{\infty} J_{2 N-1}\left(\frac{3 \omega_{Q}}{2 \omega_{r}}\right) \exp \left(-i[4 N-2] \omega_{r} t\right) \\
\cos \left(6 \int_{0}^{t} \omega_{Q}\left(t^{\prime}\right) d t^{\prime}\right) & =\sum_{N=-\infty}^{\infty} J_{2 N}\left(\frac{3 \omega_{Q}}{4 \omega_{r}}\right) \exp \left(-i 4 N \omega_{r} t\right) \\
\sin \left(6 \int_{0}^{t} \omega_{Q}\left(t^{\prime}\right) d t^{\prime}\right) & =-i \sum_{N=-\infty}^{\infty} J_{2 N-1}\left(\frac{3 \omega_{Q}}{4 \omega_{r}}\right) \exp \left(-i[4 N-2] \omega_{r} t\right) .
\end{aligned}
$$

For this crystallite, the first-order matrix elements $V_{q \pm, T \pm}^{(1)}$ for the effective Hamiltonian of Eq. 14 are given by

$$
V_{q+, T+}^{(1)}=V_{T+, q+}^{(1)}=V_{T-, q-}^{(1)}=V_{q-, T-}^{(1)}=\frac{\sqrt{5} \omega_{1}}{2} J_{0}\left(\frac{3 \omega_{Q}}{2 \omega_{r}}\right) .
$$

Consider the $+5 \mathrm{Q} \rightarrow+3 \mathrm{Q}$ coherence transfer away from rotary resonance conditions. Its efficiency depends on the magnitude of $V_{q \pm, T \pm}^{(1)}$, as shown in the Theory section. As can 
be seen in Fig. 11, the transfer depends rather sensitively on the ratio $\frac{3 \omega_{Q}}{2 \omega_{r}}$, with little or no transfer occuring when $\frac{3 \omega_{Q}}{2 \omega_{r}}$ is near a zero of the Bessel function $J_{0}(z)$. This phenomenon has been studied in the past in the context of the suppression of tunneling in two-state systems under intense radiation fields. ${ }^{41-44} \mathrm{~A}$ more detailed description connecting these phenomena will be presented in the future. Even though the size and time dependence of the quadrupolar frequency $\omega_{Q}(t)$ (Appendix A) varies for each crystallite in a powdered sample, it is a tempting possibility that by properly tuning the spinning speeds and the RF power, preferential excitations and/or conversions based on the quadrupolar frequency could be performed. 


\section{FIGURES}

FIG. 1. Partitioning of the Floquet Hamiltonian into three-dimensional subspaces. (A) Four

such subspaces, $D_{S \pm}^{N, M}$ and $D_{S \pm}^{N^{\prime}, M^{\prime}}$, are depicted in the figure. (B) Effective Hamiltonians are calculated within each subspace, treating the coupling to the other subspaces as a perturbation.

FIG. 2. The fraction of crystallites satisfying the perturbation criterion (Eq. 12) for various values of $Z$. The parameters used were $C_{Q}=3.2 \mathrm{MHz}, \eta=0$, and $\frac{\omega_{r}}{2 \pi}=20 \mathrm{kHz}$. Two thousand crystallite orientations were generated using the REPULSION technique ${ }^{36}$, from which the coefficients in Eq. 6 were calculated. (A) $Z=5$, and (B) $Z=10$.

FIG. 3. Calculation of (A) $I_{Z} \rightarrow 3 \mathrm{Q}$, (B) $I_{Z} \rightarrow 5 Q$, and (C) $+5 \mathrm{Q} \rightarrow+3 \mathrm{Q}$ coherence conversion, using the reduced effective Hamiltonian treatment given in Eqs. 19-21. The parameters used were $C_{Q}=3.2 \mathrm{MHz}, \eta=0$, and $\frac{\omega_{r}}{2 \pi}=20 \mathrm{kHz}$. Two thousand crystallite orientations were generated using the REPULSION technique ${ }^{36}$, from which the coefficients in Eq. 6 were calculated. These coefficients then were used to evaluate Eq. 17. Negative contours are drawn with a dotted line, and RR conditions are denoted by a dashed line. In (A) and (B), zeroes away from RR conditions are a result of a destructive interference over the powdered sample.

FIG. 4. Comparison of exact numerical calculation [(B), (D)] with the effective Hamiltonian treatment $[(\mathrm{A}),(\mathrm{C})]$ given by Eq. 14 for the conversion of multiple-quantum to CT coherence as a function of spin-locking power, $\frac{\omega_{1}}{2 \pi}$, and time. The parameters used were $C_{Q}=3.2 \mathrm{MHz}, \eta=0$, $\frac{\omega_{r}}{2 \pi}=20 \mathrm{kHz}$. RR conditions are denoted with a dashed line. Calculation of powder-averaged CT coherence $\left\langle C_{X}\left(\frac{\omega_{r}}{2 \pi}, t\right)\right\rangle$ from initial $[(\mathrm{A}),(\mathrm{B})]$ triple-quantum coherence, $\rho(0)=I_{X}^{2-5}$, and $[(\mathrm{C}),(\mathrm{D})]$ five-quantum coherence, $\rho(0)=I_{X}^{1-6}$. Three contour levels are shown at (A) $[0.07,0.14,0.21],(\mathrm{B})$ $[0.075,0.15,0.225]$, and at $[(\mathrm{C}),(\mathrm{D})][0.05,0.1,0.15]$. 
FIG. 5. Comparison of exact numerical calculation [(B), (D)] with the effective Hamiltonian treatment $[(\mathrm{A}),(\mathrm{C})]$ given by Eq. 14 for multiple-quantum coherence creation from $I_{Z}$ as a function of spin-locking power $\left(\frac{\omega_{1}}{2 \pi}\right)$ and time. The parameters used were $C_{Q}=3.2 \mathrm{MHz}, \eta=0, \frac{\omega_{r}}{2 \pi}=20 \mathrm{kHz}$. $\mathrm{RR}$ conditions are denoted with a dashed line. For $\rho(0)=I_{Z}$, powder-averaged triple-quantum $[(\mathrm{A}),(\mathrm{B})]\left\langle I_{Y}^{2-5}\right\rangle$ and five-quantum $[(\mathrm{C}),(\mathrm{D})]\left\langle I_{Y}^{1-6}\right\rangle$ coherences were calculated. Three contour levels, with negative contours represented with dashed lines, are shown at $[-0.55,-0.10,0.35]$.

FIG. 6. Comparison of exact numerical calculation [(B), (D)] with the effective Hamiltonian treatment $[(\mathrm{A}),(\mathrm{C})]$ given by Eq. 14 for the powder-averaged conversion of $+5 \mathrm{Q}$ coherence to $[(\mathrm{A}),(\mathrm{B})]-3 \mathrm{Q}$ coherence and $[(\mathrm{C}),(\mathrm{D})]+3 \mathrm{Q}$ coherence as a function of spin-locking power $\left(\frac{\omega_{1}}{2 \pi}\right)$ and time. The parameters used were $C_{Q}=3.2 \mathrm{MHz}, \eta=0$, and $\frac{\omega_{r}}{2 \pi}=20 \mathrm{kHz}$. RR conditions are denoted with a dashed line. Three contour levels are shown at $[(\mathrm{A}),(\mathrm{B})][0.05,0.10,0.15]$ and at $[(\mathrm{C}),(\mathrm{D})][0.15,0.30,0.45]$.

FIG. 7. Comparison of exact numerical calculation $[(\mathrm{B}),(\mathrm{D}),(\mathrm{F})]$ with the effective Hamiltonian treatment $[(\mathrm{A}),(\mathrm{C}),(\mathrm{E})]$ given by Eq. 14 for the conversion of $+5 \mathrm{Q}$ coherence to $+3 \mathrm{Q}$ coherence as a function of spin-locking power $\left(\frac{\omega_{1}}{2 \pi}\right)$ and time for three different crystallite orientations. The parameters used were $C_{Q}=3.2 \mathrm{MHz}, \eta=0$, and $\frac{\omega_{r}}{2 \pi}=20 \mathrm{kHz}$. RR conditions are denoted with a dashed line. $[(\mathrm{A}),(\mathrm{B})] \beta=10^{\circ},[(\mathrm{C}),(\mathrm{D})] \beta=45^{\circ}$, and $[(\mathrm{E}),(\mathrm{F})] \beta=90^{\circ}$. Three contour levels are shown, with dashed contours indicating negative values, with [(A),(B)] $[0.2,0.4,0.6],[(\mathrm{C}),(\mathrm{D})][0.3,0.6,0.9]$, and $[(\mathrm{E}),(\mathrm{F})][0.25,0.5,0.75]$. Good agreement is obtained between the theory and simulations for crystallites with $\beta=45^{\circ}$ and $90^{\circ}$, whereas less agreement is obtained for $\beta=10^{\circ}$. 
FIG. 8. Contour plots of the maximum intensity of experimental 1D MQMAS absolute-value spectra, as functions of the RF amplitude and pulse width of the RR pulse. (A) $+5 \mathrm{Q}$ coherence $\rightarrow$ $+3 \mathrm{Q}$ coherence conversion. The maximum efficiency for $+5 \mathrm{Q}$ coherence $\rightarrow+3 \mathrm{Q}$ coherence transfer occurs away from the rotary resonance conditions of $\omega_{1}=\frac{2}{3} n \omega_{r}$ for $n=1$ or 2 . (B) $+3 \mathrm{Q}$ coherence $\rightarrow+1 \mathrm{Q}$ coherence conversion.

FIG. 9. Exact numerical calculation of $+5 \mathrm{Q} \rightarrow+3 \mathrm{Q}$ coherence transfer for a powdered sample, including the effects of the second-order quadrupolar coupling. Twelve-hundred crystallite orientations were chosen. The parameters used were $C_{Q}=3.2 \mathrm{MHz}, \eta=0$, and $\frac{\omega_{r}}{2 \pi}=20 \mathrm{kHz}$. RR conditions are denoted by a dashed line. Contours are drawn at the levels $[0.07,0.14,0.21,0.28]$. Fig. $6[(\mathrm{C}),(\mathrm{D})]$ (exact simulation with no second-order quadrupolar coupling) shows the same basic features, although the intensities of the conversion are smaller here, due to dephasing by the second-order quadrupolar coupling.

FIG. 10. Comparison of the unimodal Floquet treatment with the bimodal Floquet treatment for a spin $I=3 / 2$. In unimodal Floquet theory, the states $|C \pm, N\rangle$ are split by energy $2 \omega_{1}$, where $\omega_{1}$ is the RF field strength. In bimodal Floquet theory, the states $|C \pm, N\rangle$ are dressed by the $|M\rangle$ RF "oscillator" state, which is used instead to keep track of the energy splitting.

FIG. 11. Exact numerical calculation of the effects of the ratio $\frac{\omega_{Q}}{\omega_{r}}$ on the $+5 \mathrm{Q} \rightarrow+3 \mathrm{Q}$ coherence transfer efficiency for the single crystallite given by $[\alpha, \beta, \gamma]=\left[0^{\circ}, 90^{\circ}, 0^{\circ}\right]$. Here $\frac{\omega_{r}}{2 \pi}=20 \mathrm{kHz}$, and an $\mathrm{RF}$ field strength of $\frac{\omega_{1}}{2 \pi}=8 \mathrm{kHz}$ was chosen in order to be away from any RR condition. The coupling between the states $| \pm 5 / 2\rangle$ and $| \pm 3 / 2\rangle$ is proportional to the Bessel function $J_{0}\left(Z_{r}\right)$, where $Z_{r}=\frac{3 \omega_{Q}}{4 \omega_{r}}$. (A) $\frac{\omega_{Q}}{2 \pi}=430 \mathrm{kHz}, J_{0}\left(Z_{r}\right)=0.1404$, (B) $\frac{\omega_{Q}}{2 \pi}=50 \mathrm{kHz}, J_{0}\left(Z_{r}\right)=-0.4014$, and (C) $\frac{\omega_{Q}}{2 \pi}=157.22 \mathrm{kHz}, J_{0}\left(Z_{r}\right)=-8.6 \times 10^{-6}$. The coherence transfer in (C) is practically negligible over this time scale, since the corresponding $Z_{r}$ is nearly a zero of the Bessel function $J_{0}(z)$. 


\section{TABLES}

TABLE I. Phase cycles for the $+3 \mathrm{Q} \rightarrow+1 \mathrm{Q}$ and $+5 \mathrm{Q} \rightarrow+3 \mathrm{Q}$ conversion experiments. The labels $\phi_{1,2,3,4, r}$ denote the phase of the pulse or receiver. The phases are given in degrees. Subscripts indicate the number of repetitions of the phase cycle in the parentheses, brackets, or braces. There are 96 and 640 steps in the $+3 \mathrm{Q} \rightarrow+1 \mathrm{Q}$ and $+5 \mathrm{Q} \rightarrow+3 \mathrm{Q}$ conversion and phase cycles, respectively. experiment phases phase list

$$
\begin{aligned}
& 3 \mathrm{Q} \rightarrow 1 \mathrm{Q} \text { con. } \phi_{1} \quad(0,30,60,90,120,150,180,210,240,270,300,330)_{8} \\
& \phi_{2} \quad(0)_{96} \\
& \phi_{3} \quad(0)_{12},(45)_{12},(90)_{12},(135)_{12},(180)_{12},(225)_{12},(270)_{12},(315)_{12} \\
& \phi_{r} \quad\left[(0,270,180,90)_{3},(90,0,270,180)_{3},(180,90,0,270)_{3},(270,180,90,0)_{3}\right]_{2} \\
& 5 \mathrm{Q} \rightarrow 3 \mathrm{Q} \text { con. } \phi_{1} \quad(0,18,36,54,72,90,108,126,144,162, \\
& 180,198,216,234,252,270,288,306,324,342)_{32} \\
& \phi_{2} \quad\left[(0)_{20},(90)_{20},(180)_{20},(270)_{20}\right]_{8} \\
& \phi_{3} \quad(0)_{80},(45)_{80},(90)_{80},(135)_{80},(180)_{80},(225)_{80},(270)_{80},(315)_{80} \\
& \phi_{4} \quad(0)_{640} \\
& \phi_{r} \quad\left\{\left[(0,270,180,90)_{5},(180,90,0,270)_{5}\right]_{2},\left[(90,0,270,180)_{5},(270,180,90,0)_{5}\right]_{2},\right. \\
& \left.\left[(180,90,0,270)_{5},(0,270,180,90)_{5}\right]_{2},\left[(270,180,90,0)_{5},(90,0,270,180)_{5}\right]_{2}\right\}_{2}
\end{aligned}
$$




\section{REFERENCES}

${ }^{1}$ J. H. Baltisberger et al., J. Am. Chem. Soc. 118, 7209 (1996).

${ }^{2}$ I. Farnan et al., Nature 358, 31 (1992).

${ }^{3}$ J. F. Stebbins, J. V. Oglesby, and S. K. Lee, Chem. Geol. 174, 63 (2001).

${ }^{4}$ K. E. Vermillion, P. Florian, and P. J. Grandinetti, J. Chem. Phys. 108, 7274 (1998).

${ }^{5}$ K. T. Mueller, J. H. Baltisberger, E. W. Wooten, and A. Pines, J. Phys. Chem. 96, 7001 (1992).

${ }^{6}$ P. Zhao, P. S. Neuhoff, and J. F. Stebbins, Chem. Phys. Lett. 344, 325 (2001).

${ }^{7}$ K. H. Lim and C. P. Grey, J. Am. Chem. Soc. 122, 9768 (2000).

${ }^{8}$ C. A. Fyfe, J. Skibsted, and W. Schweiger, Inorg. Chem. 40, 5906 (2001).

${ }^{9}$ A. Llor and J. Virlet, Chem. Phys. Lett. 152, 248 (1988).

${ }^{10}$ A. Samoson, E. Lippmaa, and A. Pines, Mol. Phys. 65, 1013 (1988).

${ }^{11}$ K. T. Mueller et al., J. Magn. Reson. 86, 470 (1990).

${ }^{12}$ L. Frydman and J. S. Harwood, J. Am. Chem. Soc. 117, 5367 (1995).

${ }^{13}$ A. J. Vega, J. Magn. Reson. 96, 50 (1992).

${ }^{14}$ A. J. Vega, Solid State Nucl. Magn. Reson. 1, 17 (1992).

${ }^{15}$ G. Wu, D. Rovnyak, and R. G. Griffin, J. Am. Chem. Soc. 118, 9326 (1996).

${ }^{16}$ W. Sun, J. T. Stephen, L. D. Porter, and Y. Wu, J. Magn. Reson. Ser. A 116, 181 (1995).

17 S. M. DePaul et al., J. Phys. Chem. B 101, 3240 (1997).

${ }^{18}$ M. Baldus, D. Rovnyak, and R. G. Griffin, J. Chem. Phys. 112, 5902 (2000).

19 T. Vosegaard, P. Florian, D. Massiot, and P. J. Grandinetti, J. Chem. Phys. 114, 4618 
(2001).

${ }^{20}$ J. D. Walls, K. H. Lim, and A. Pines, J. Chem. Phys. 116, 79 (2002).

${ }^{21}$ C. Cohen-Tannoudji, J. Dupont-Roc, and G. Grynberg, Atom-Photon Interactions (John Wiley \& Sons, Inc., Berlin, 1992).

${ }^{22}$ S. Vega and A. Pines, J. Chem. Phys. 66, 5624 (1978).

${ }^{23}$ S. Vega, J. Chem. Phys. 68, 5518 (1978).

${ }^{24}$ Z. H. Gan and P. J. Grandinetti, Chem. Phys. Lett. (accepted).

${ }^{25}$ J. H. Shirley, Phys. Rev. 138, 979 (1965).

${ }^{26}$ A. Schmidt and S. Vega, J. Chem. Phys. 96, 2655 (1992).

${ }^{27}$ E. Vinogradov, P. K. Madhu, and S. Vega, Chem. Phys. Lett. 329, 207 (2000).

${ }^{28}$ T. O. Levante, M. Baldus, B. H. Meier, and R. R. Ernst, Mol. Phys. 86, 1195 (1995).

${ }^{29}$ Y. Zur, M. H. Levitt, and S. Vega, J. Chem. Phys. 66, 5293 (1983).

${ }^{30}$ S. Ding and C. A. McDowell, Mol. Phys 95, 979 (1998).

${ }^{31}$ I. Shavitt and L. Redmon, J. Chem. Phys. 73, 5711 (1980).

${ }^{32}$ E. Vinogradov, P. K. Madhu, and S. Vega, J. Chem. Phys. 115, 8983 (2001).

${ }^{33}$ R. Ramesh and M. S. Krishnan, J. Chem. Phys. 115, 5967 (2001).

${ }^{34}$ Solid-state NMR Spectroscopy, edited by M. Duer (Blackwell Science Ltd., Oxford, 2002).

${ }^{35}$ M. Mehring, Principles of High Resolution NMR in Solids, 2nd ed. (Spinger-Verlag, Berlin, 1983).

${ }^{36}$ M. Bak and N. C. Nielsen, J. Magn. Reson. 125, 132 (1997).

${ }^{37}$ S. P. Brown and S. Wimperis, J. Magn. Reson. 128, 42 (1997). 
${ }^{38}$ S. E. Ashbrook, S. P. Brown, and S. Wimperis, Chem. Phys. Lett. 288, 509 (1998).

${ }^{39}$ A. Jerschow, J. W. Logan, and A. Pines, J. Magn. Reson. 149, 268 (2001).

${ }^{40}$ J. W. Logan, J. T. Urban, A. Jerschow, and A. Pines, in preparation .

${ }^{41}$ J. C. A. Barata and W. F. Wreszinski, Phys. Rev. Lett. 84, 2112 (2000).

${ }^{42}$ M. Frasca, J. Optics B-Quantum S. O. 3, S15 (2001).

${ }^{43}$ A. Santana, J. M. G. Llorente, and V. Delgado, J. Phys. B - At. Mol. Opt. 34, 2371 (2001).

${ }^{44}$ V. Delgado and J. M. G. Llorente, J. Phys. B - At. Mol. Opt. 33, 5403 (2000). 
J. D. Walls et. al Figure 1

(A)

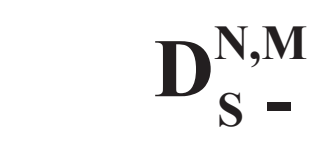

$\left|\mathrm{q}^{-}, \mathrm{N}, \mathrm{M}\right\rangle$

$|\mathrm{T}-\mathrm{N}, \mathrm{M}\rangle$

$\mathbf{D}_{\mathrm{S}+}^{\mathrm{N}, \mathbf{M}}$

$|\mathrm{c}-\mathrm{N}+\mathrm{n}, \mathrm{M}-1\rangle$

$\mathbf{D}_{\mathrm{s}-}^{\mathrm{N}^{\prime}, \mathrm{M}^{\prime}}$

$\mathbf{D}_{\mathbf{S}^{+}}^{\mathrm{N}^{\prime}, \mathbf{M}^{\prime}}$
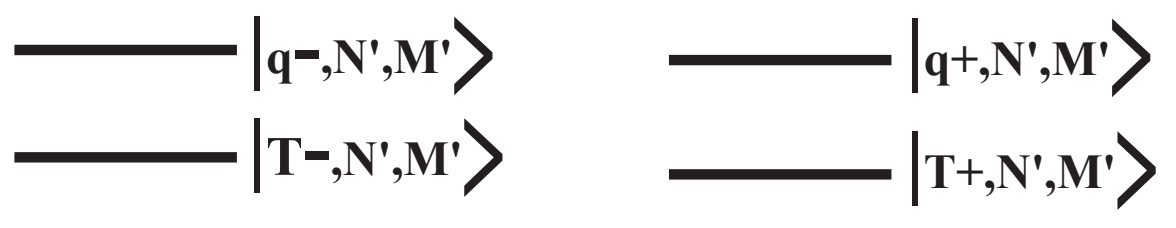

$\widehat{\mathrm{H}}_{\mathrm{F}}=$ $\left|\mathrm{C}-\mathrm{N}^{\prime}+\mathrm{n}, \mathrm{M}^{\prime}-1\right\rangle$

$\left|C^{+}, N^{\prime}-n, M^{\prime}+1\right\rangle$

(B)

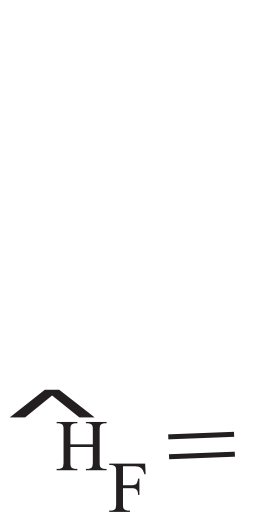

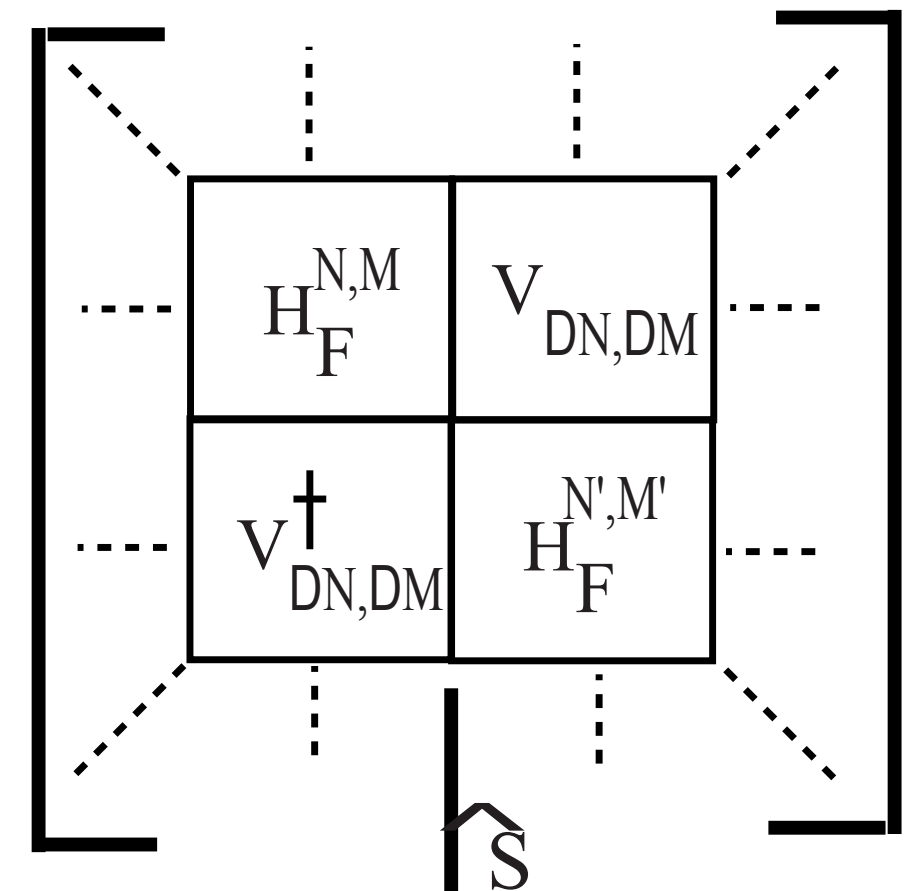

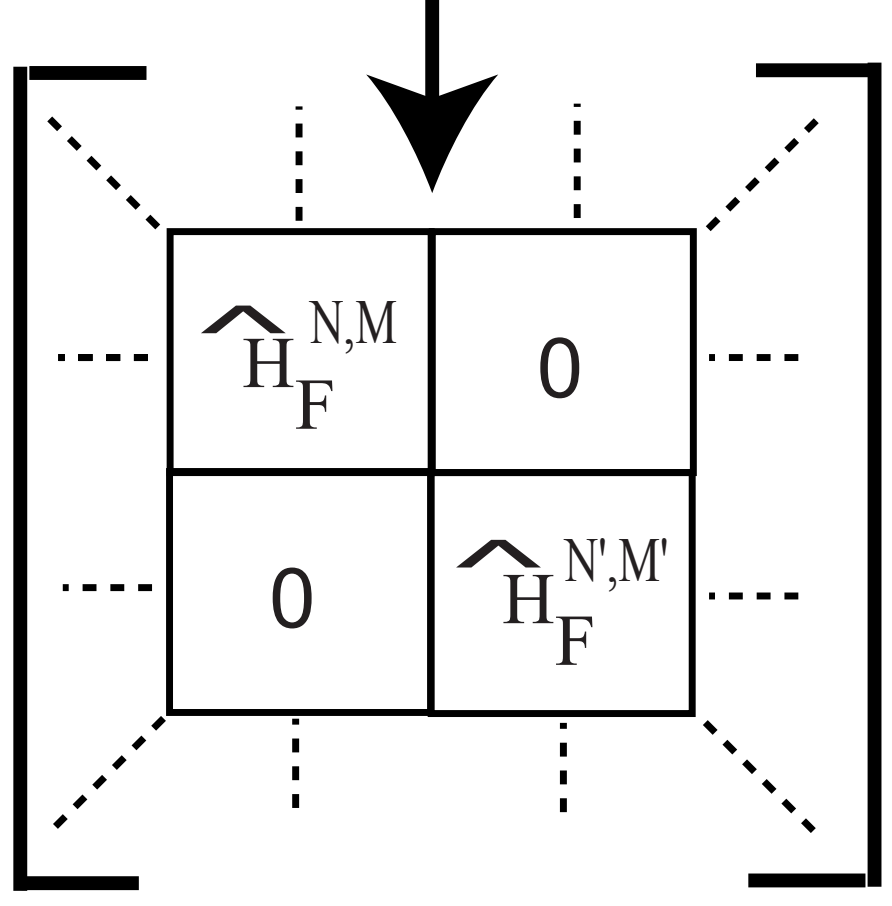


J. D. Walls et. al Figure 2
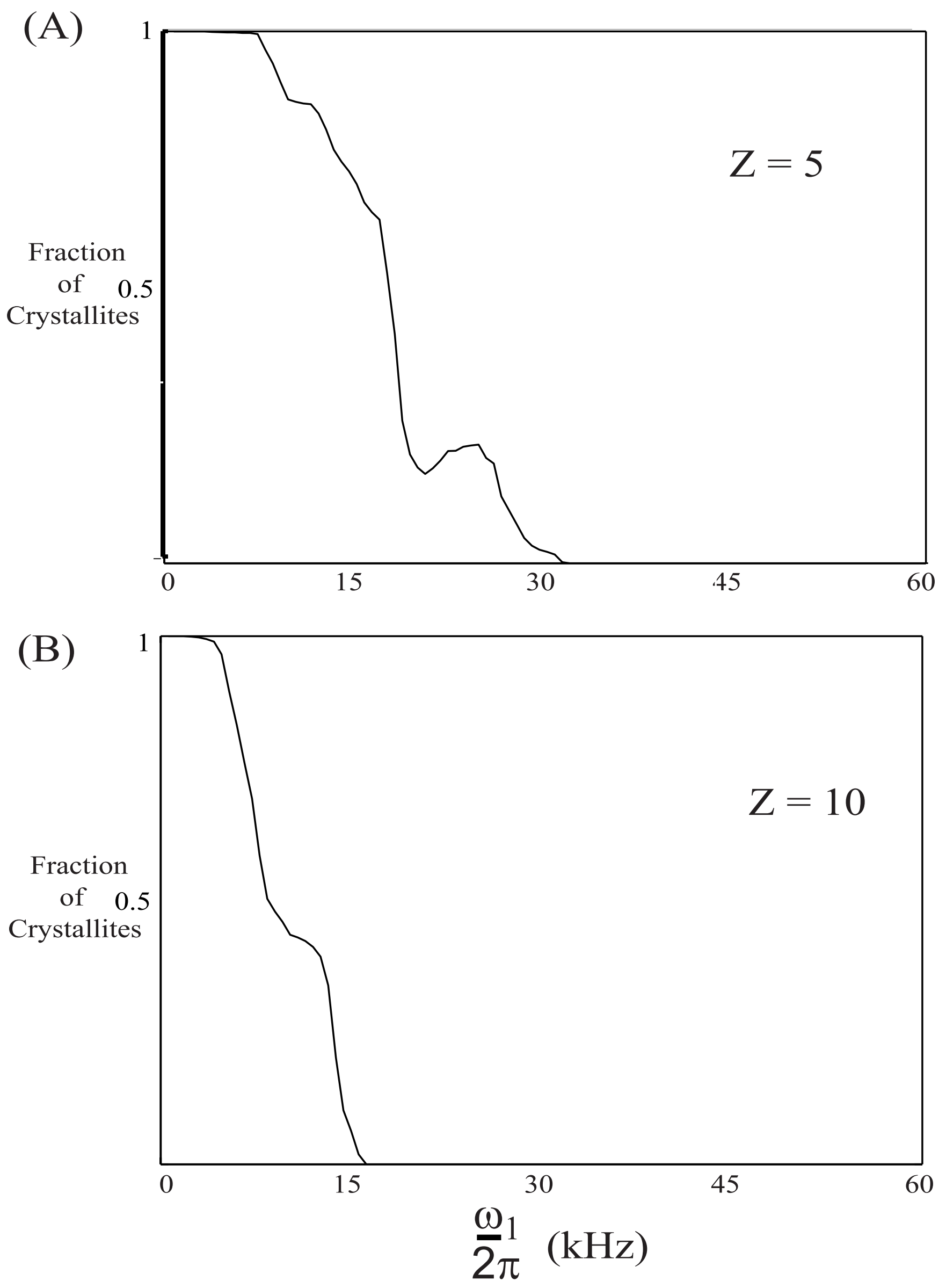


\section{J. D. Walls et. al Figure 3}
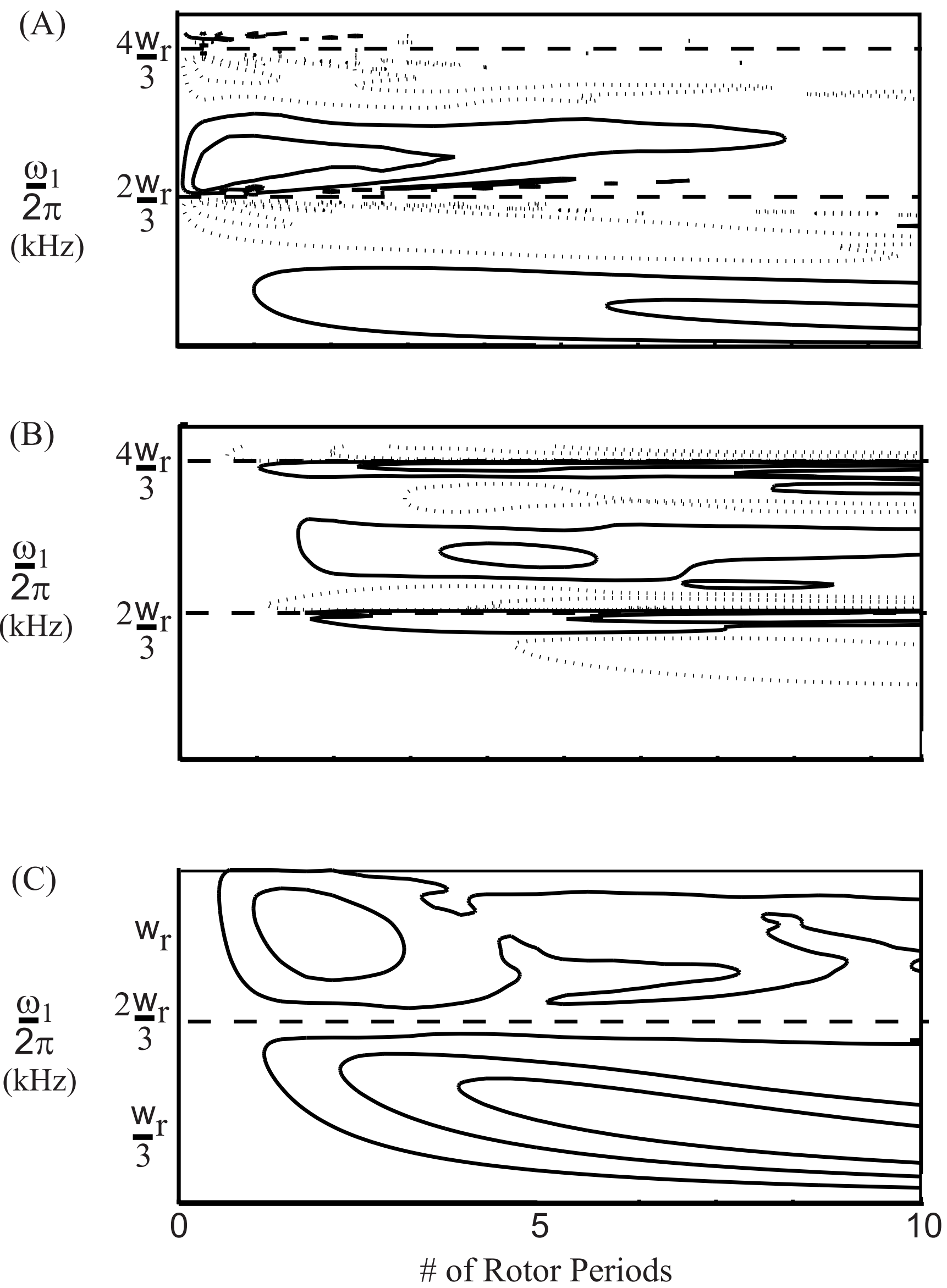


\section{J. D. Walls et. al Figure 4}

(A)

(B)
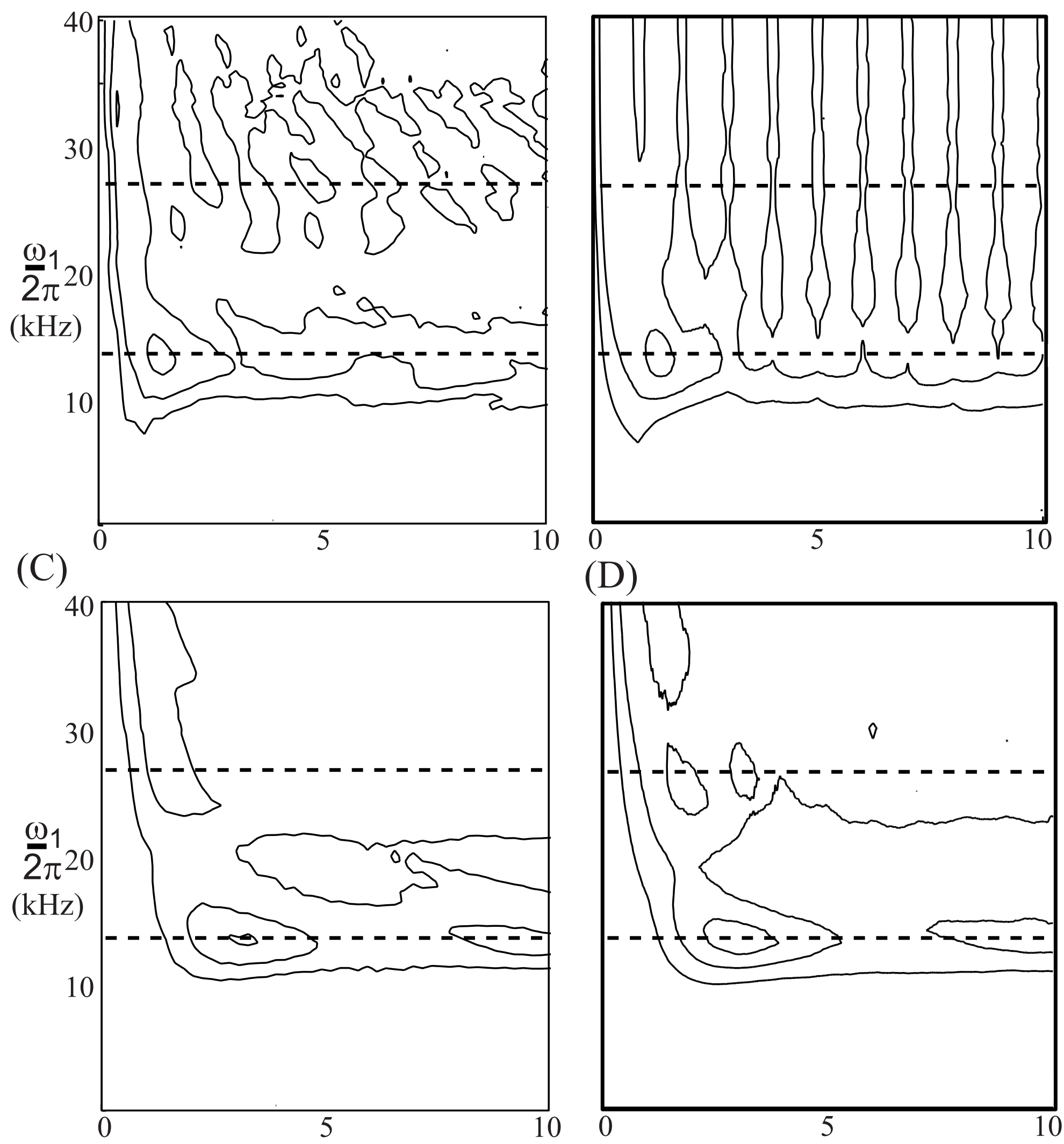

\# of Rotor Periods

\# of Rotor Periods 


\section{J. D. Walls et. al Figure 5}

(A)
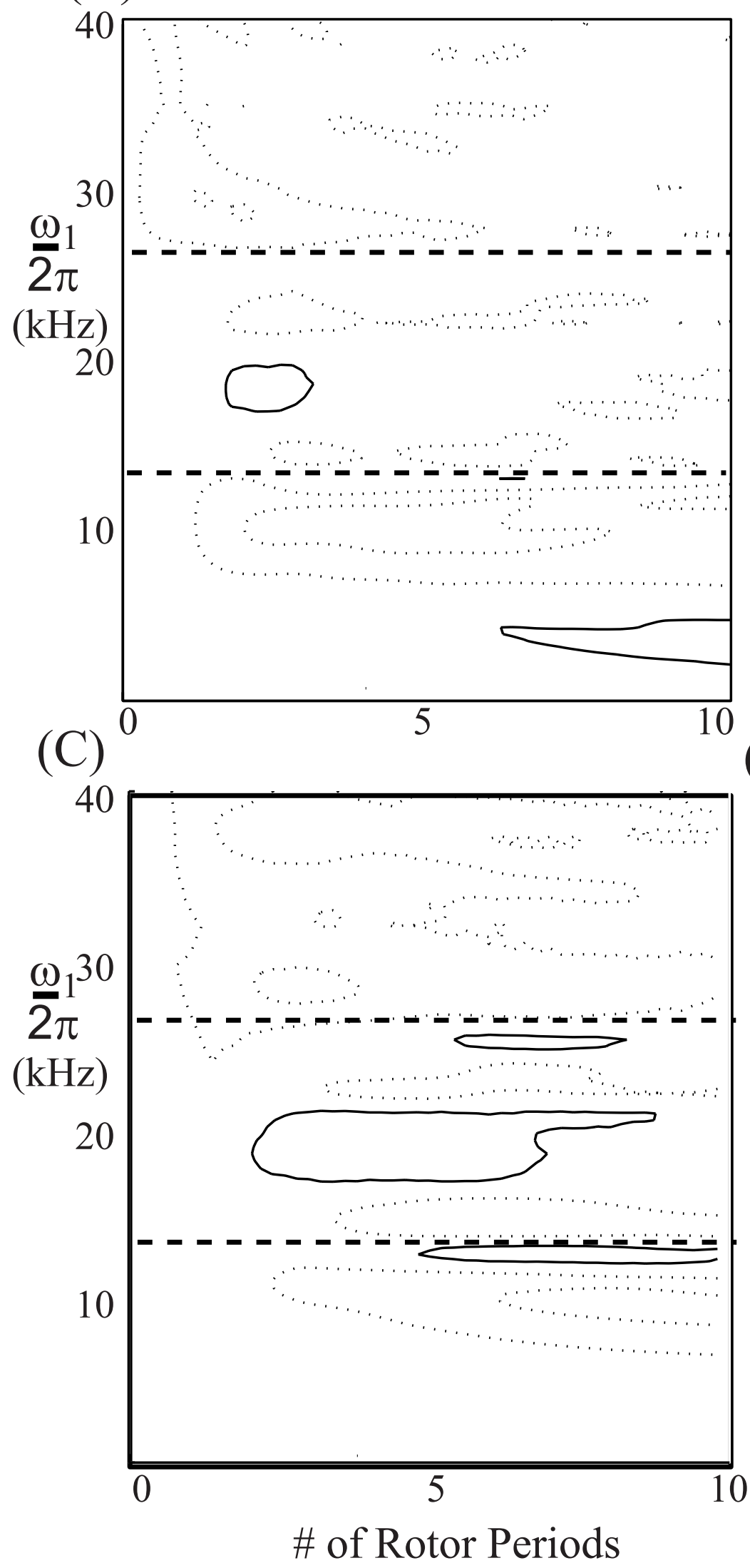

(B)
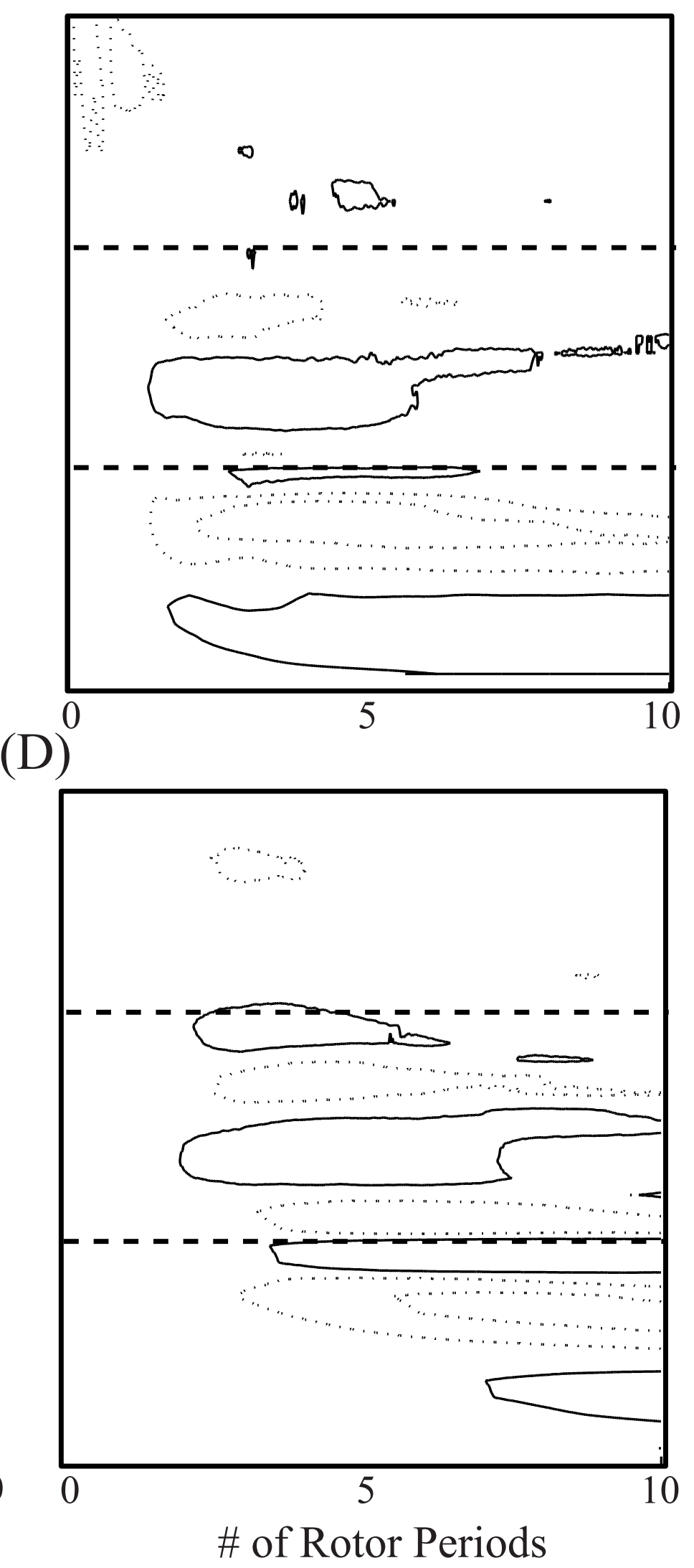


\section{J. D. Walls et. al Figure 6}

(A)
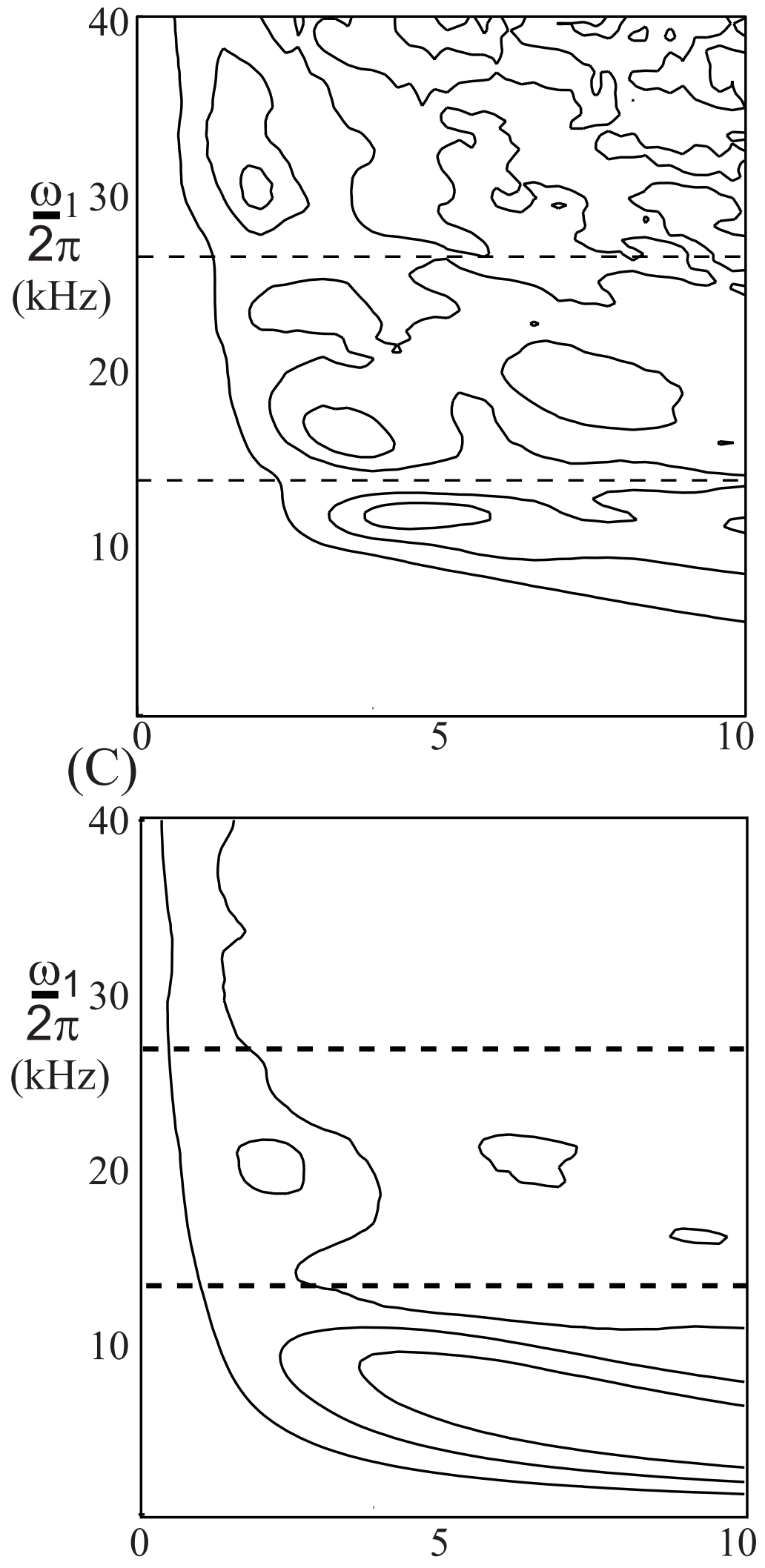

\# of Rotor Periods
(B)

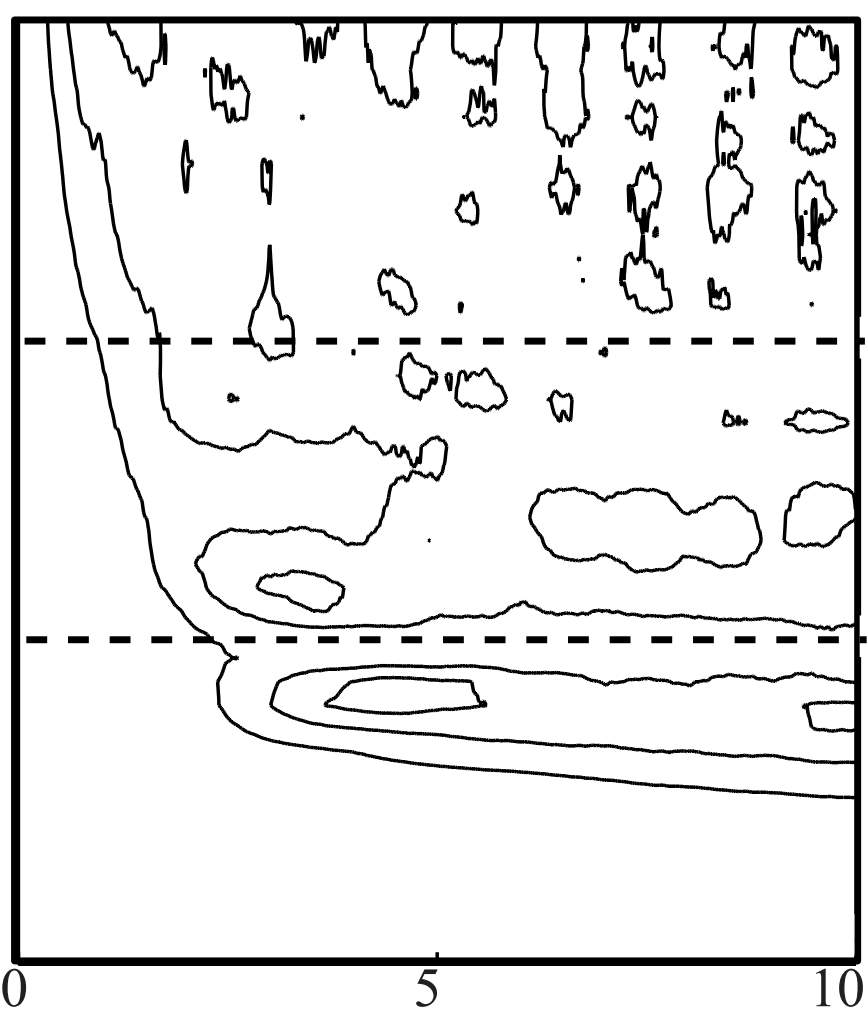

(D)

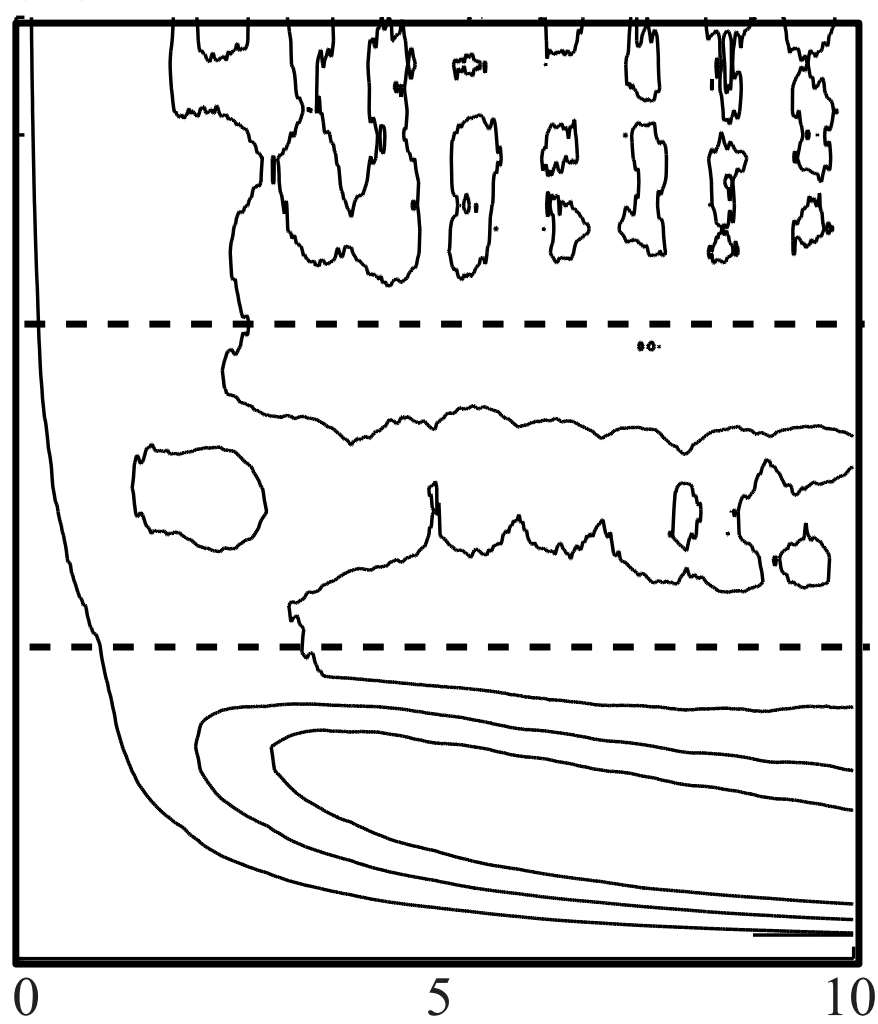

\# of Rotor Periods 


\section{J. D. Walls et. al Figure 7}

(A)
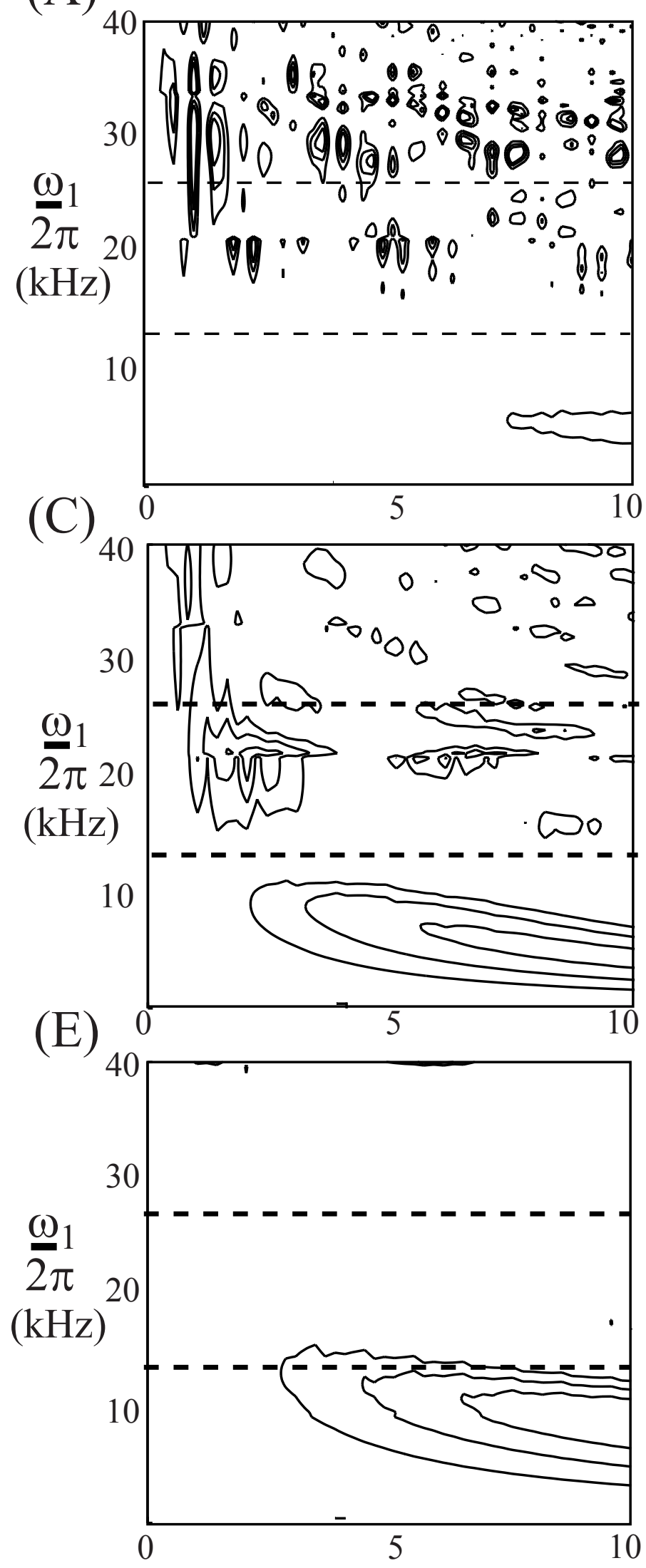

\# of Rotor Periods
(B)
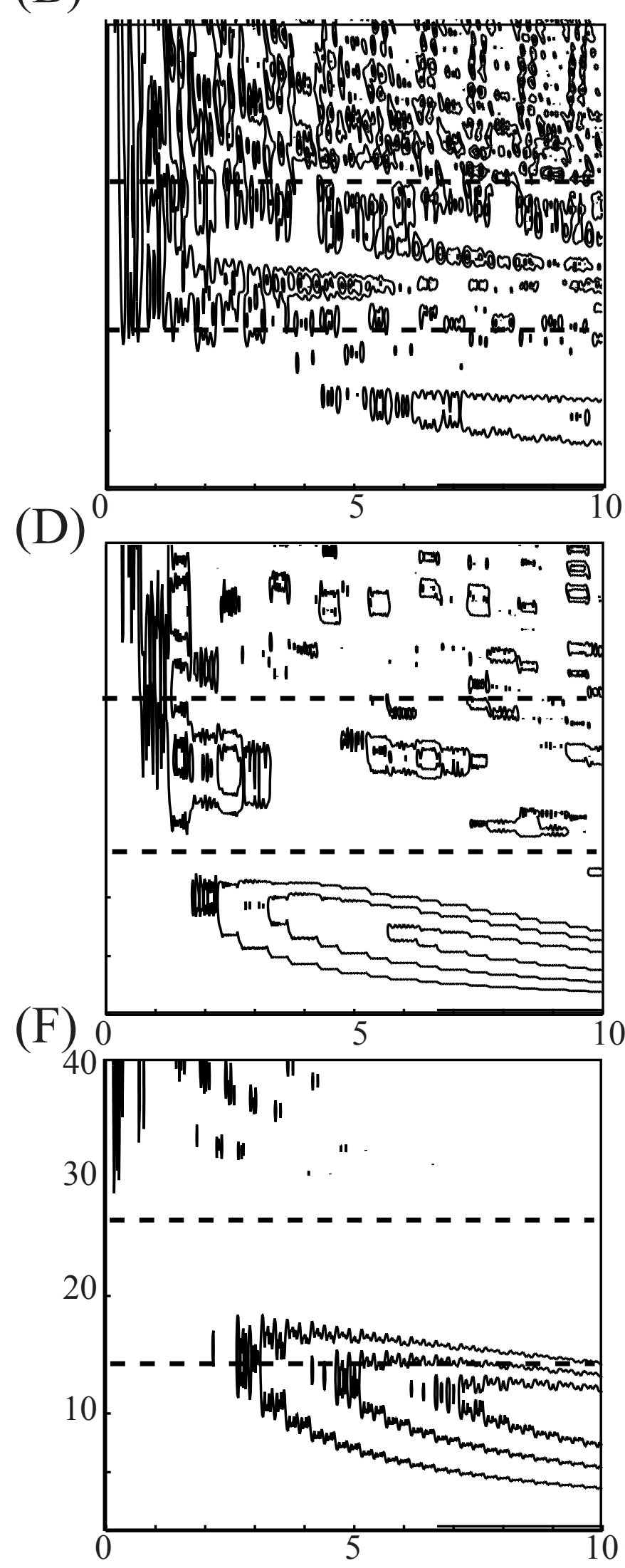

\# of Rotor Periods 


\section{J. D. Walls et. al Figure 8}
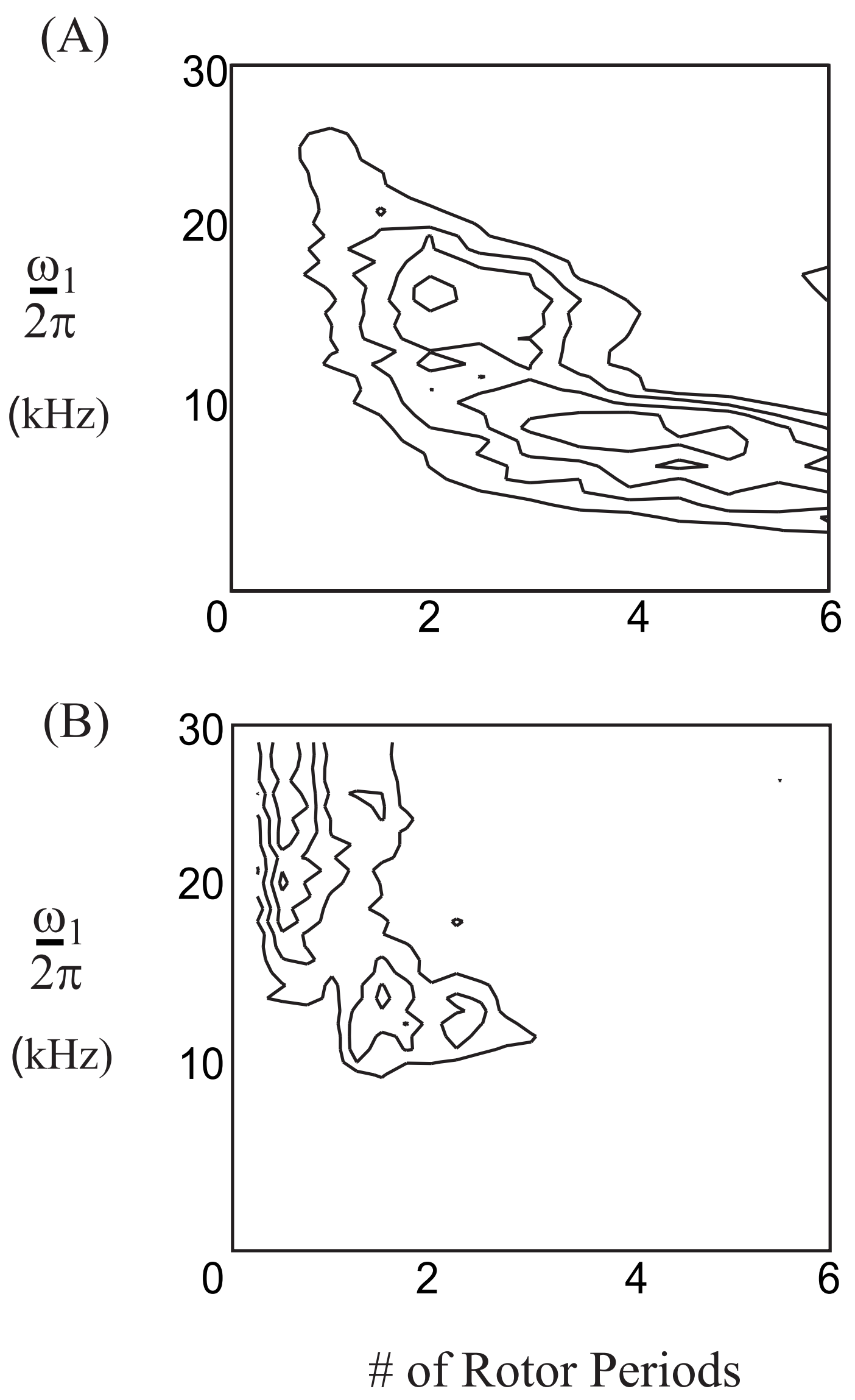
J. D. Walls et. al Figure 9

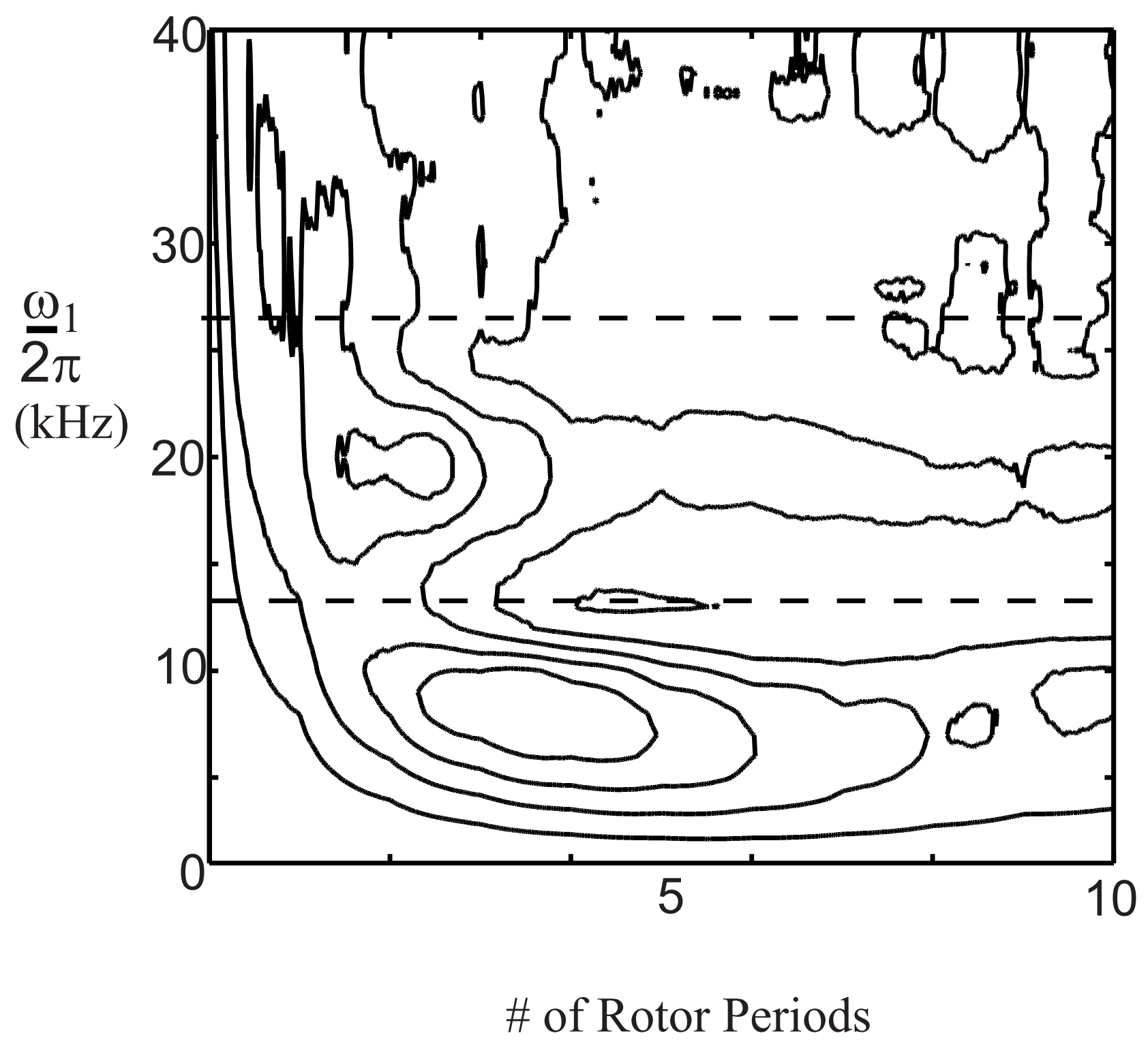




\section{J. D. Walls et. al Figure 10}

Unimodal Floquet States

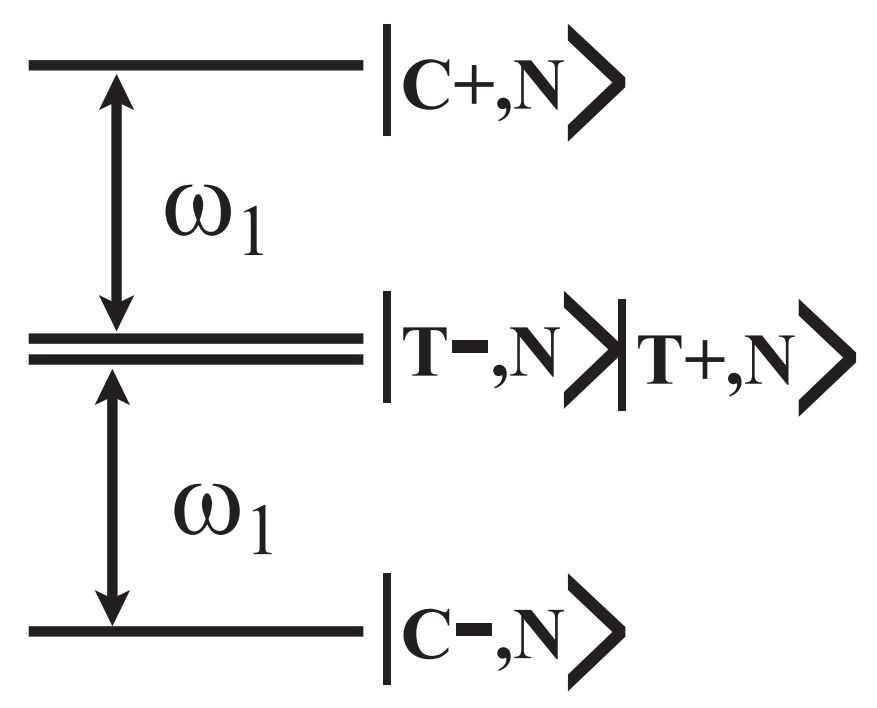

Bimodal Floquet States

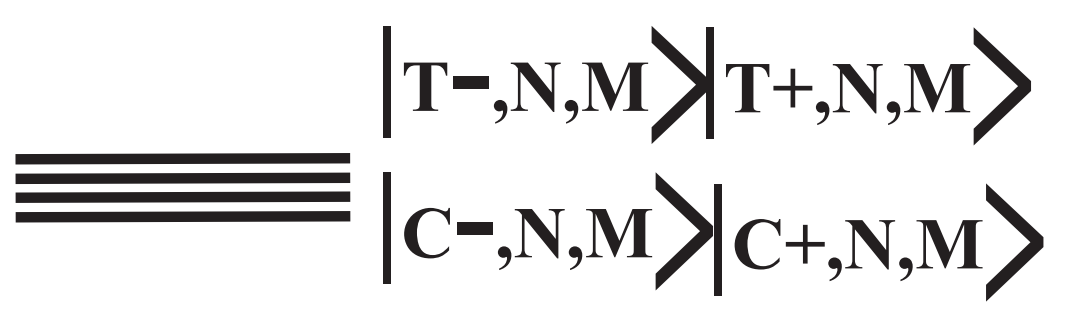


J. D. Walls et. al Figure 11

(A)
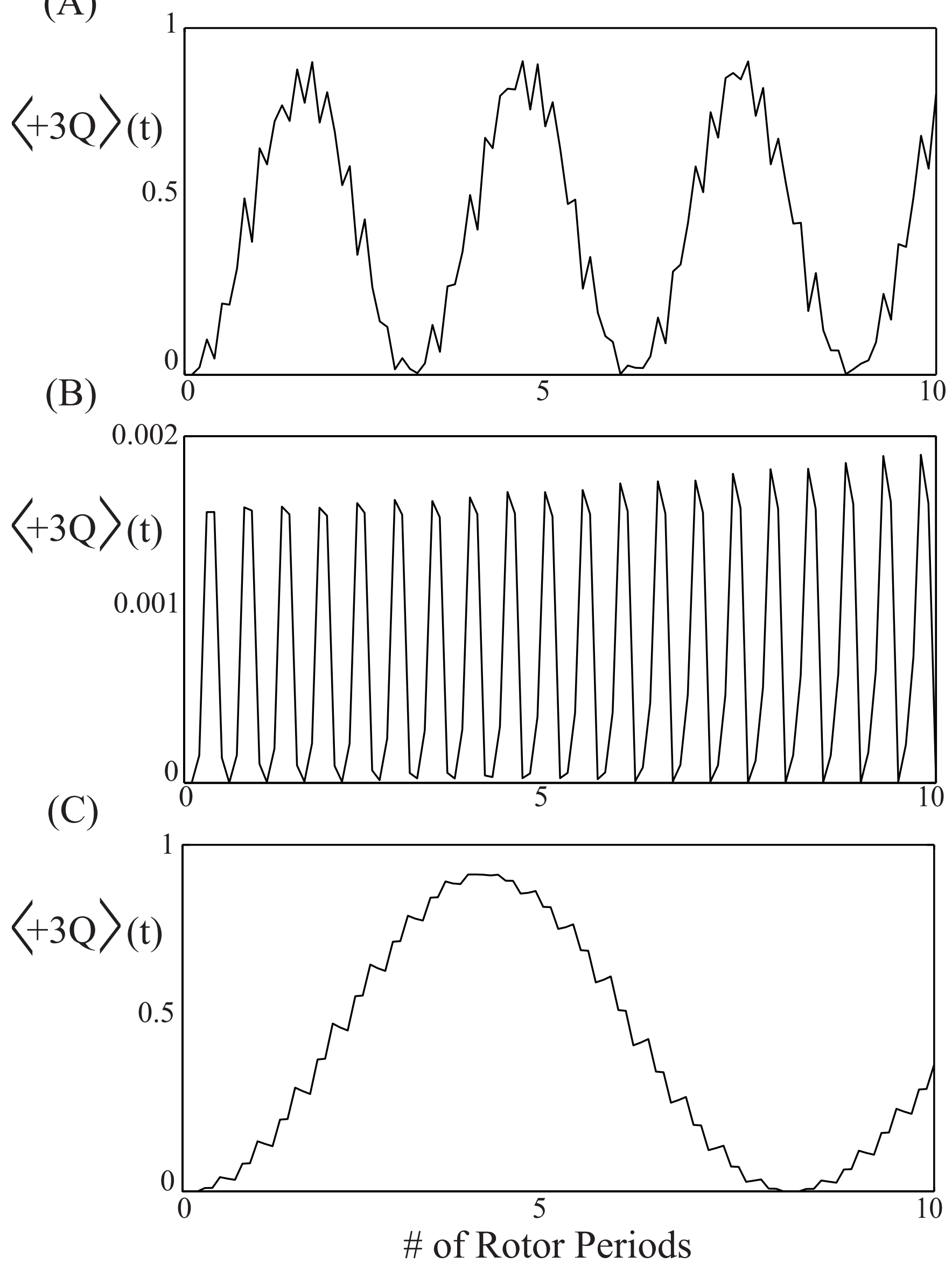